\title{
Application of a multi-layer systems toxicology framework for in vitro assessment of the biological effects of Classic Tobacco e-liquid and its corresponding aerosol using an e-cigarette device with MESH $^{\mathrm{Tm}}$ technology
}

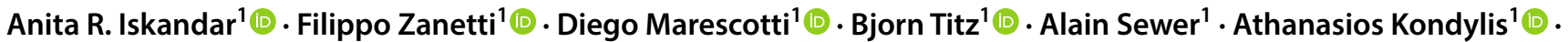 \\ Patrice Leroy ${ }^{1}(1) \cdot$ Vincenzo Belcastro $^{1}$ - Laura Ortega Torres ${ }^{1}$ - Stefano Acali ${ }^{1}$. Shoaib Majeed ${ }^{1}$. Sandro Steiner ${ }^{1}$. \\ Keyur Trivedi ${ }^{1}$. Emmanuel Guedj ${ }^{1}$. Celine Merg ${ }^{1}$ - Thomas Schneider ${ }^{1}$. Stefan Frentzel ${ }^{1}$. Florian Martin ${ }^{1}$. \\ Nikolai V. Ivanov ${ }^{1}$ (D) Manuel C. Peitsch ${ }^{1}$ D $\cdot$ Julia Hoeng $^{1}$ (D)
}

Received: 2 July 2019 / Accepted: 2 September 2019 / Published online: 7 September 2019

(c) The Author(s) 2019

\begin{abstract}
We previously proposed a systems toxicology framework for in vitro assessment of e-liquids. The framework starts with the first layer aimed at screening the potential toxicity of e-liquids, followed by the second layer aimed at investigating the toxicity-related mechanism of e-liquids, and finally, the third layer aimed at evaluating the toxicity-related mechanism of the corresponding aerosols. In this work, we applied this framework to assess the impact of the e-liquid MESH Classic Tobacco and its aerosol compared with that of cigarette smoke (CS) from the 3R4F reference cigarette. In the first layer, we evaluated the cytotoxicity profile of the MESH Classic Tobacco e-liquid (containing humectants, nicotine, and flavors) and its Base e-liquid (containing humectant and nicotine only) in comparison with total particulate matter (TPM) of 3R4F CS using primary bronchial epithelial cell cultures. In the second layer, the same culture model was used to explore changes in specific markers using high-content screening assays to identify potential toxicity-related mechanisms induced by the MESH Classic Tobacco and Base e-liquids beyond cell viability in comparison with the 3R4F CS TPM-induced effects. Finally, in the third layer, we compared the impact of exposure to the MESH Classic Tobacco or Base aerosols with 3R4F CS using human organotypic air-liquid interface buccal and small airway epithelial cultures. The results showed that the cytotoxicity of the MESH Classic Tobacco liquid was similar to the Base liquid but lower than 3R4F CS TPM at comparable nicotine concentrations. Relative to 3R4F CS exposure, MESH Classic Tobacco aerosol exposure did not cause tissue damage and elicited lower changes in the mRNA, microRNA, and protein markers. In the context of tobacco harm reduction strategy, the framework is suitable to assess the potential-reduced impact of electronic cigarette aerosol relative to CS.
\end{abstract}

Keywords E-cigarettes $\cdot$ Transcriptomics $\cdot$ Systems toxicology $\cdot$ High-content screening $\cdot$ Airway epithelial

\section{Introduction}

Anita R. Iskandar, Filippo Zanetti, and Diego Marescotti contributed equally to this work.

Electronic supplementary material The online version of this article (https://doi.org/10.1007/s00204-019-02565-9) contains supplementary material, which is available to authorized users.

Anita R. Iskandar

anita.iskandar@pmi.com

1 Philip Morris International R\&D, Philip Morris Products S.A., Quai Jeanrenaud 5, 2000 Neuchâtel, Switzerland
For decades, the effort to reduce harm caused by smoking has focused on preventing smoking initiation and promoting smoking cessation; however, a consistent increase in the rate of cessation from 1991 to 2010 failed to be demonstrated (Zhu et al. 2012). The tobacco harm reduction approach, which is designed to reduce health risks associated with tobacco smoking, but may involve the continued use of nicotine (Cox and Dawkins 2018), has been increasingly proposed as a promising complementary strategy to accelerate the decline in smoking prevalence and to reduce 
the overall smoking-related population harm (WHO 2008). Modified risk tobacco products (Family Smoking Prevention and Tobacco Control Act 2009) are relevant to this effort; they should significantly reduce the harm and risk of smoking-related disease for individual smokers and improve the health of the population as a whole.

A recent study stated that electronic cigarettes (EC) "may be a unique harm reduction innovation for smoking relapse prevention" and that vaping can be a long-term substitute for cigarette smoking with "substantial implications for tobacco harm reduction" (Notley et al. 2018). Nonetheless, the safety of EC use is highly debated. ECs "are electrical devices that generate an aerosol from a liquid" (Bals et al. 2019). The liquid, generally referred to as e-liquid, is typically composed of humectants (e.g., propylene glycol [PG] and vegetable glycerin $[\mathrm{VG}]$ ) and flavors, with or without nicotine (Grana et al. 2014; NAS 2018). With more than 8000 flavors now available on the market (Bals et al. 2019) and around 242 new flavors added every month (Tierney et al. 2016), the selection of e-liquids adequate for use with ECs should be driven by relevant toxicological analysis.

An earlier proposal promoted the selection of e-liquid ingredients with certain purity criteria and to avoid ingredients known to be carcinogenic, mutagenic, and reprotoxic (Costigan and Meredith 2015). The authors also suggested the exclusion of known respiratory sensitizers. A later proposal recommended the selection of ingredients based on their potential respiratory allergens (Costigan and LopezBelmonte 2017). Finally, a recent article suggested the use of Genomic Allergen Rapid Detection to classify and differentiate flavors based on their sensitizing potentials (Stevenson et al. 2019). In addition, our group has proposed a multi-layer systems toxicology framework for in vitro assessment of e-liquids that is meant to complement the battery of classical assays for mutagenicity and genotoxicity testing (Iskandar et al. 2016). Briefly, the first layer of the framework is aimed at screening e-liquids for potential toxicity using relevant 2-dimensional (2D) primary human cell culture systems. This is followed, in the second layer, by toxicity-related mechanistic investigations of selected e-liquids using culture systems similar to the one used in the first layer. Finally, the third layer of the framework focuses on toxicity-related mechanistic investigation of the corresponding aerosols using 3-dimensional (3D) airway culture systems grown at the air-liquid interface (ALI). This proposed framework advocates the use of in vitro test systems relevant for the inhalation route.

In the present work, we assessed whether the MESH Classic Tobacco e-liquid elicited lower biological effects than CS in vitro using the multi-layer systems toxicology framework outline above (Iskandar et al. 2016). The first and second layers of the assessment investigated the toxicity profiles of both the MESH Classic Tobacco e-liquid (containing
PG, VG, nicotine, and flavors) and its corresponding Base e-liquid (containing only PG, VG, and nicotine without flavors) in comparison with the total particulate matter (TPM) of CS from the 3R4F reference cigarette using submerged 2D cultures of primary normal human bronchial epithelial (NHBE) cells. The third layer of the assessment focused on investigating the biological impact of the aerosols generated by the MESH EC device from both MESH Classic Tobacco and Base e-liquids in comparison with the impact of 3R4F CS using 3D ALI primary human buccal and small airway epithelial culture models. The aerosol was generated with an EC device based on the $M E S H$ technology (P4M3 generation 1.0, Philip Morris International).

Our choice of testing the impact of EC exposure on 2D bronchial and 3D small airway epithelial cultures may be obvious given the importance of the lung as the primary target organ upon inhalation exposure. However, studies have shown that gene expression changes in the upper respiratory tract (nasal epithelium) overlap with changes detected in the lower respiratory tract (bronchial epithelium) (Steiling et al. 2008). Furthermore, a recent study using multiscale computational fluid-particle dynamics model combined with a physiologically based toxicokinetic model also reported that EC aerosol particle deposition occurs not only in the respiratory tract, but also in the mouth (Haghnegahdar et al. 2018). Therefore, in the present work, we also tested the impact of the EC aerosol exposure on buccal epithelial cultures (part of the third layer of the framework). This approach is aligned with the National Institute of Dental and Craniofacial Research (NIDCR) Strategic Plan 2014-2019 and its initiatives in assessing the effects of EC aerosols on oral health. NIDCR recently noted the lack of studies investigating the effects of EC aerosol exposure on periodontal epithelial cells (NIDCR 2018).

\section{Materials and methods}

\section{D NHBE cells}

NHBE cells were purchased from Lonza (Basel, Switzerland) and were derived from a healthy 60-year-old Caucasian male donor who was consuming alcohol (but not in a diseased state), with no history of smoking (Lot number 0000140733). The provider certified that the cells tested negative for mycoplasma, bacteria, yeast, and fungi, and that HIV-1, hepatitis B, and hepatitis $C$ were not detected in the cell lot. For all experiments described here, we used cells between passages 5 and 8 . Cells were cultured in a bronchial epithelial cell medium (Bullet Kit, catalog no CC-3170, Lonza, Cologne, Germany) as previously described (Gonzalez-Suarez et al. 2017). 


\section{E-liquid formulations}

Two different formulations of e-liquid were tested using the primary 2D NHBE cells (Table 1).

\section{R4F CS TPM}

TPM of 3R4F CS was prepared by collecting the mainstream smoke from the 3R4F reference cigarette on Cambridge glass fiber filters (44 $\mathrm{mm}$ diameter) following a previously published protocol (Gonzalez-Suarez et al. 2016). The mainstream smoke of 6 cigarettes was trapped in 2 separate glass fiber filters followed by extraction in a plastic vessel. The first filter was extracted with $5 \mathrm{~mL}$ ethanol, and the second filter was extracted with the first crude extract.

\section{Real-time cell analysis (RTCA)-based data generation and analysis}

2D NHBE cells were seeded into E-Plate View 96-well tissue culture plates (ACEA Biosciences, San Diego, CA, USA) at a density of $7.2 \times 10^{3}$ cells per well in $100 \mu \mathrm{L}$ of culture medium and incubated at $37 \pm 1{ }^{\circ} \mathrm{C}$ in a humidified incubator with $5.0 \pm 0.5 \% \mathrm{CO}_{2}$ for $24 \pm 1 \mathrm{~h}$. Cells were then exposed to different dilutions of each test solution (Fig. 1, First-Layer Assessment). A positive control (Triton X-100; T8787, Sigma-Aldrich, St. Louis, MO, USA) and negative control (cell culture medium) were included in each experiment. A set of 7 independent experiments was conducted to test 3R4F CS TPM, and 4 independent experiments were conducted to test MESH Classic Tobacco and Base liquids.

Impedance was measured continuously using an xCELLigence RTCA ${ }^{\circledR}$ system (ACEA Biosciences, San Diego, CA, USA) from the timepoint of cell seeding until $24 \mathrm{~h}$ of exposure to the MESH Classic Tobacco liquid and its Base liquid. The resulting values were normalized to both the negative control and the positive control within the plate (2-point normalization) to obtain the relative cell viability (see Supplementary Materials and Methods).

Table 1 Composition of test e-liquids

\begin{tabular}{llllll}
\hline Test e-liquid & \multicolumn{3}{l}{ Content $(\mathrm{w} / \mathrm{w}, \%)$} \\
\cline { 2 - 6 } & PG (\%) & VG $(\%)$ & Nicotine (\%) & Flavors & $\begin{array}{l}\text { Other } \\
\text { (e.g., } \\
\text { water) }\end{array}$ \\
\hline $\begin{array}{l}\text { MESH classic } \\
\begin{array}{l}\text { tobacco } \\
\text { liquid }\end{array}\end{array}$ & 39 & 39 & 1.8 & $\checkmark$ & $\checkmark$ \\
\begin{tabular}{l} 
Base liquid \\
\hline
\end{tabular} & 39 & 39 & 1.8 & - & $\checkmark$ \\
\hline
\end{tabular}

\section{Generation of high-content screening (HCS) data}

2D NHBE cells were seeded into black, clear-bottom 96-well tissue culture plates at a density of 37,500 cells per $\mathrm{cm}^{2}(12,000$ cells per well) in $100 \mu \mathrm{L}$ of culture medium. The cells were incubated for $24 \mathrm{~h}$ in the culture medium and then exposed (in 2 replicate wells) to increasing concentrations of the tested solutions (Fig. 1, Second-Layer Assessment). The corresponding flavorless solution (Base liquid) was included as a reference. The cells were exposed for 4 and $24 \mathrm{~h}$ before performing the HCS assays (cell membrane permeability, cytochrome c release, DNA damage [pH2AX], glutathione (GSH) content, oxidative stress [ROS], stress kinase [c-Jun phosphorylation]) using various dyes and following previously published methods (Gonzalez-Suarez et al. 2016; Marescotti et al. 2016). A set of 3-8 independent experiments was conducted. A more detailed description of the protocol is provided in the Supplementary Materials and Methods.

\section{HCS data processing}

HCS assays ( $N=3-8$ independent experiments) generate multiple fluorescence readouts that are measured simultaneously. The quantification of fluorescence images was stored in the database linked to the GladiaTOX package (Belcastro et al. 2019), which extends the ToxCast analysis pipeline package (Filer et al. 2017). As a quality check, positive controls were first normalized against the corresponding vehicle (cell culture medium) and analyzed. Data (endpoints) that did not pass the quality criteria were masked. Raw data that passed the quality check were normalized to vehicle using the following equation:

$N(i)=\log _{2}\left(\frac{i}{V e h}\right)$,

where $i$ is the measured raw signal value of a well, and Veh is the median of the measured signal values for the vehicle wells on a plate.

Dose-response curves were fitted on the normalized data using three function families: constant, Hill, and gain-loss functions. The best-fitting model of the three function families (minimizing the Akaike information criteria) was retained, and a minimum effective concentration (MEC), which was defined as the intersection of the fitted curve with the noise band, was calculated (Supplementary Fig. 1). The noise band was computed as three times the baseline median absolute deviation of vehicle responses. MEC values are not available for the constant model (the modeled activity never intersects the noise band). Missing MEC values were replaced by the value for the maximum tested concentration except if all replicates showed a constant signal. 


\section{First Layer Assessment}

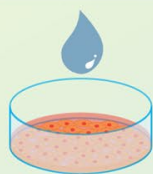

2D NHBE

\section{$24 \mathrm{~h}$}

Incubation with MESH Classic Tobacco liquid (containing PG, G, nicotine and flavors)

- Incubation with Base liquid (containing PG, G, and nicotine without flavors)

- Incubation with the total particulate matter (TPM) of 3R4F reference cigarette smoke

\section{ENDPOINTS}

Cytotoxicity measurement using real-time cell analysis (RTCA)

\section{Second Layer Assessment}

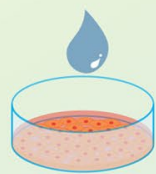

2D NHBE
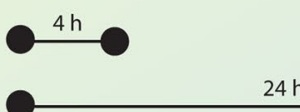

- Incubation with MESH Classic Tobacco liquid (containing PG, G, nicotine and flavors)

- Incubation with Base liquid (containing PG, G, and nicotine without flavors)

- Incubation with the TPM of 3R4F reference cigarette smoke
ENDPOINTS

High content screening assays:

- Cell membrane permeability

- Cytochrome c release

- DNA damage ( $\mathrm{pH} 2 \mathrm{AX})$

- Glutathione content

- Oxidative stress (ROS)

- Stress kinase (c-Jun)

\section{Third Layer Assessment}

Test Aerosol

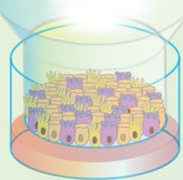

3D Human

Organotypic

Epithelial Cultures

(Buccal and Small Airway)

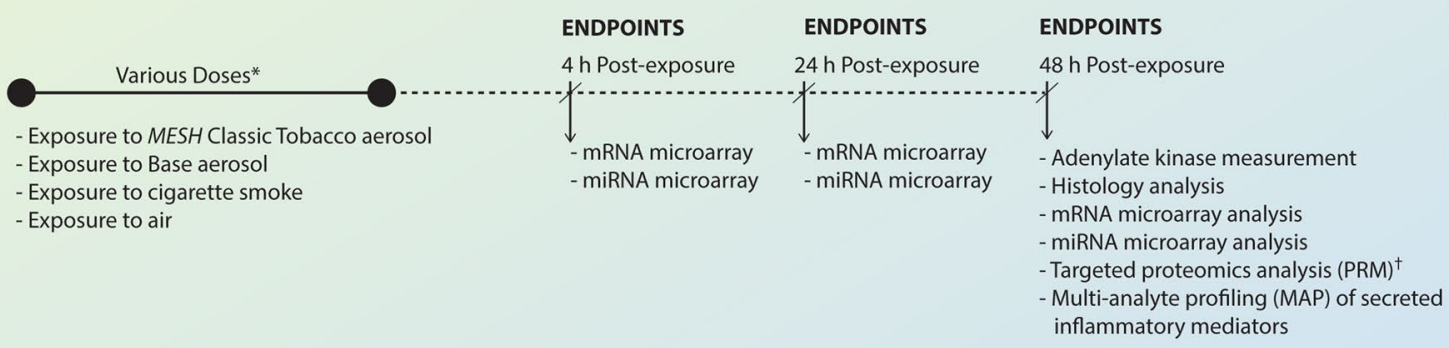

Fig. 1 Experimental procedures and biological endpoints. Applying the first layer of the assessment framework (Iskandar et al. 2016), we examined the cytotoxicity effects of MESH Classic Tobacco and Base liquids compared with those of 3R4F CS TPM using 2D primary NHBE cultures (upper panel). Subsequently, in the second layer of the assessment, toxicity-related mechanistic evaluation of $M E S H$ Classic Tobacco and Base liquids, compared with 3R4F CS TPM, was conducted using high-content screening (HCS) assays on 2D

\section{D human organotypic ALI buccal and small airway epithelial cultures}

Organotypic human buccal epithelial (EpiOral ${ }^{\mathrm{TM}}$, MatTek Corp., Ashland, MA, USA) and small airway epithelial (SmallAir ${ }^{\mathrm{TM}}$, Epithelix. Geneva, Switzerland) cultures were used; each was reconstituted from the primary cells of a single donor (Table 2). We chose to use a single donor to primary NHBE cultures (middle panel). Finally, the third layer of the assessment investigated the biological impact of the MESH Classic Tobacco and Base aerosols compared with that of 3R4F CS using 3D human buccal and small airway epithelial cultures. *Various doses tested are given in Table 3. For the 3D buccal epithelial cultures, PRM was generated from samples collected at $24 \mathrm{~h}$ post-exposure. $\mathrm{G}$, glycerol; p2HAX, phosphorylated H2A histone family member X; PG, propylene glycol; ROS, reactive oxygen species

reduce the influence of donor-to-donor variability and thus increase the statistical power to identify potential exposure effects, although we acknowledge that the results are limited to a donor-specific response. Buccal cultures were approximately 2 weeks after reconstruction before being used for the exposure experiment. Small airway cultures were approximately 5.5 weeks after air lift before being used for the exposure experiment. The cells were maintained according to
Table 2 Human organotypic ALI buccal and small airway culture models

\begin{tabular}{lll}
\hline Description & EpiOral & SmallAir \\
\hline Cell origin & Human buccal epithelial cells & Human bronchiolar epithelial cells \\
Culture model & 3D organotypic epithelial monoculture & 3D organotypic epithelial monoculture \\
Donor profile & 40 years, male & 47 years, female \\
Donor smoking status & Nonsmoker & Nonsmoker \\
Donor pathology status & No pathology reported & No pathology reported \\
\hline
\end{tabular}


previously published protocols (Iskandar et al. 2018; Zanetti et al. 2018). After exposure, the medium was not changed until the cultures were collected for various endpoint measurements (up to $48 \mathrm{~h}$ post-exposure [PE]).

\section{Generation of 3R4F CS and EC aerosols}

Mainstream CS was generated from 3R4F reference cigarettes (Kentucky Tobacco Research \& Development Center; University of Kentucky, Lexington, KY, USA) using a 30-port carousel smoking machine (SM2000; Philip Morris, International). Test aerosols were generated from the MESH Classic Tobacco or Base liquids using an EC device with MESH technology (P4M3 generation 1.0, Philip Morris International) in combination with single-programmable syringe pumps. The $M E S H$ technology maintains the temperature for the heater between 200 and $220{ }^{\circ} \mathrm{C}$ rather than varying the temperature depending on the puff strength. The particle sizes of 3R4F CS, MESH Classic Tobacco aerosol, and Base aerosol were similar; they were measured before entering the exposure system as a quality control following a previously published protocol (Iskandar et al. 2019); data are given Supplementary Table 1.

\section{Exposure setup for the ALI cultures}

We conducted a set of 3 experimental phases using buccal cultures and a set of 3 using small airway cultures. In each phase, in which 1 specific culture batch was used, 3 independent exposure experiments were conducted. Thus, a total of 9 independent exposure experiments were performed. The positioning of cultures (assigned for a given endpoint) in the exposure plate was changed from experiment-to-experiments to reduce allocation bias. VITROCELL ${ }^{\circledR}$ exposure systems (VITROCELL ${ }^{\circledR}$ 24/48; VITROCELL Systems GmbH, Waldkirch, Germany) were used to house the cultures during the exposure. In 1 exposure system, up to 48 cultures can be simultaneously exposed to smoke or aerosols. In each plate, we included the cultures that were exposed only to air (controls), thus generating paired samples (exposed vs. airexposed control samples). Various endpoints were assessed following exposures (Fig. 1, Third-Layer Assessment). In addition to biological cultures, we included phosphate-buffered saline (PBS) in the exposure chamber that was used to measure the concentrations of deposited nicotine $(100 \mu \mathrm{L}$ PBS/well).

A 30-port carousel smoking machine (Philip Morris International) was connected to 1 dedicated VITROCELL ${ }^{\circledR}$ $24 / 48$ exposure system. A total of 4 puffs/minute were taken consecutively from 2 cigarettes placed in the 30 -port carousel. The puff generation from 1 cigarette was conducted for a puff volume of $55 \mathrm{~mL}$, a puff duration of $2 \mathrm{~s}$, and a puff interval of $30 \mathrm{~s}$ (Health Canada 1999), with an exhaust time of $8 \mathrm{~s}$ and with $100 \%$ blocking of the filter ventilation.

The single-programmable syringe pumps (for the generation of MESH Classic Tobacco and Base aerosols) were connected to another dedicated VITROCELL ${ }^{\circledR} 24 / 48$ exposure system. Two EC devices of the same kind (i.e., containing MESH Classic Tobacco liquid or Base liquid) were connected to 1 pump; a pinch valve installed between the cartridges and the pump alternated the activation of the 2 devices, generating 1 puff every $15 \mathrm{~s}$ (a total of 4 puffs/minute were generated). The puff generation from 1 EC device was conducted for a puff volume of $55 \mathrm{~mL}$, a puff duration of $3 \mathrm{~s}$, and a puff interval of $30 \mathrm{~s}$ (CORESTA 2015), with an exhaust time of $8 \mathrm{~s}$.

Doses of 3R4F CS, MESH Classic Tobacco aerosol, and Base aerosol applied to the cultures are summarized in Table 3. The concentrations of 3R4F CS (112-puff exposure $=28$-min exposure) were chosen based on the observations in our previous studies, where we could detect a wide range of toxic response, from subtoxic to toxic (Iskandar et al. 2018; Zanetti et al. 2018). Because this is a comparative assessment in which the impact of MESH Classic Tobacco or Base aerosols was compared with that of 3R4F $\mathrm{CS}$, the doses chosen for MESH Classic Tobacco and Base aerosols were those resulting in a similar and/or higher nicotine concentrations deposited in the exposure chamber compared with 3R4F CS exposure (Table 3).

\section{Histology analysis on ALI epithelial cultures}

Histological sections were obtained from cultures harvested $48 \mathrm{~h} \mathrm{PE}$, because morphological alterations would occur at later timepoints following exposure and after molecular changes took place (Castro et al. 2008). The processing of the organotypic cultures was conducted following previously published protocols (Iskandar et al. 2018; Zanetti et al. 2018).

\section{Adenylate kinase release assay}

Adenylate kinase activity in the basolateral medium of the cultures was measured from different cultures at $48 \mathrm{~h}$ PE using ToxiLight ${ }^{\mathrm{TM}}$ bioassay kit (Lonza, Basel, Switzerland), according to the manufacturer's instructions. A luminescence signal was measured using a FLUOStar Omega reader (BMG LABTECH GmbH, Ortenberg, Germany). For each of the three experimental repetitions/phases (i.e., each culture batch), the values of the luminescence signal were normalized to the mean value of the positive (Triton X-100-treated cultures) and negative (untreated) control samples as previously described (Iskandar et al. 2018). The mean values (from a total of 
Table 3 Smoke and aerosol exposure doses

\begin{tabular}{|c|c|c|c|c|}
\hline & $\begin{array}{l}\text { Smoke or aerosol concentra- } \\
\text { tion }(\%) \text { fed into the exposure } \\
\text { chamber }^{\mathrm{a}}\end{array}$ & $\begin{array}{l}\text { Duration of } \\
\text { exposure } \\
(\min )\end{array}$ & $\begin{array}{l}\text { Total puff } \\
\text { numbers }\end{array}$ & $\begin{array}{l}\text { Median concentration of deposited nicotine in } \\
\text { the exposure chamber }(\mu \mathrm{g} \text { nicotine } / \mathrm{mL} \text { PBS })^{\mathrm{c}}\end{array}$ \\
\hline \multicolumn{5}{|l|}{ Buccal } \\
\hline Air (control for CS) & $0^{\mathrm{b}}$ & 28 & 112 & NA \\
\hline 3R4F CS concentration 1 & 24 & 28 & 112 & 14 \\
\hline 3R4F CS concentration 2 & 69 & 28 & 112 & 92 \\
\hline Air (control for EC aerosol) & $0^{\mathrm{b}}$ & 28 & 112 & NA \\
\hline$M E S H$ classic tobacco aerosol & 100 & 28 & 112 & 52 \\
\hline Base aerosol & 100 & 28 & 112 & 33 \\
\hline Air (control for EC aerosol) & $0^{\mathrm{b}}$ & 112 & 224 & NA \\
\hline MESH classic tobacco aerosol & 100 & 112 & 224 & 159 \\
\hline Base aerosol & 100 & 112 & 224 & 143 \\
\hline \multicolumn{5}{|l|}{ Small airway } \\
\hline Air (control for CS) & $0^{\mathrm{b}}$ & 28 & 112 & NA \\
\hline 3R4F CS concentration 1 & 7 & 28 & 112 & 4 \\
\hline 3R4F CS concentration 2 & 13 & 28 & 112 & 10 \\
\hline Air (control for the EC aerosol) & $0^{\mathrm{b}}$ & 7 & 28 & NA \\
\hline MESH classic tobacco aerosol & 100 & 7 & 28 & 3 \\
\hline Base aerosol & 100 & 7 & 28 & 4 \\
\hline Air (control for the EC aerosol) & $0^{\mathrm{b}}$ & 28 & 112 & NA \\
\hline MESH classic tobacco aerosol & 100 & 28 & 112 & 53 \\
\hline Base aerosol & 100 & 28 & 112 & 54 \\
\hline
\end{tabular}

NA not applicable

${ }^{a}$ Values refer to the smoke or aerosol concentration fed into the VITROCELL ${ }^{\circledR}$ 24/48 Dilution/Distribution Module

${ }^{\mathrm{b}}$ Controls refer to $100 \%$ air administered to the culture on the same exposure plate

${ }^{\mathrm{c}}$ Concentrations of nicotine were measured using liquid chromatography coupled with tandem mass spectrometry (LC-MS/MS) using PBS located in the wells $(100 \mu \mathrm{L} /$ well $)$ within the exposure chamber

9 independent exposure experiments) of the normalized relative luminescence units were then reported in figures as percentages.

\section{Measurement of secreted inflammatory mediators from ALI epithelial cultures}

MAP of inflammatory mediators secreted into the basolateral medium of cultures was performed using commercially available Milliplex panels (Merck Millipore, Burlington, MA, USA) with Luminex ${ }^{\circledR}$ xMAP $^{\circledR}$ Technology (Luminex, Austin, TX, USA)-based analysis, according to the manufacturer's instructions and following previously published protocols (Iskandar et al. 2018; Zanetti et al. 2018). The samples were randomized prior to the analysis. The data were log-transformed, and the geometric means were used to calculate the fold changes (FC) of the mediators (exposed vs. air-exposed samples), $N=9$ independent exposure experiments. A more detailed description is provided in the Supplementary Materials and Methods.

\section{Targeted proteomics by PRM}

Targeted proteomics by PRM was conducted at the $24 \mathrm{~h}$ PE time for the ALI buccal epithelial cultures $(N=9$ independent exposure experiments), and at the $48 \mathrm{~h}$ PE time for the ALI small airway epithelial cultures $(N=9$ independent exposure experiments) following a previous published protocol (Iskandar et al. 2017). The timepoint collection for each culture type was decided based on the previous studies in which we detected greater changes in the protein levels following exposure (data not shown). Samples were randomized prior to the analysis. A more detailed description is provided in the Supplementary Materials and Methods.

Raw files from the PRM acquisition were analyzed with SpectroDive (version 8.0 Biognosys AG). Ion chromatograms for the endogenous peptides and the corresponding stable isotope-labeled reference peptides were extracted for all measured transitions [the list of transitions was reported in a previous publication (Iskandar et al. 2017)] using the software vendor's default settings. For quantification, the area under the curve (AUC) intensities of all transitions were 
summed, and ratios of AUC sums of the endogenous and corresponding reference peptide signal were calculated.

For the statistical analysis, a linear model was fitted for each exposure and the respective sham group, including the experimental repetition as a covariate to account for the pairing between exposure and sham groups (Smyth 2004). The obtained raw $p$ values (without empirical Bayes moderation, corresponding to a paired $t$ test), were adjusted across protein markers using the Benjamini-Hochberg false discovery rate (FDR) method. Differentially expressed proteins were defined as those with an FDR-adjusted $p$ value $<0.05$.

\section{RNA isolation and array analyses from ALl epithelial cultures}

Total RNA, including miRNA, was isolated from the epithelial cultures ( $N=9$ independent exposure experiments) using a previously published method (Iskandar et al. 2018; Zanetti et al. 2018). Samples were randomized before processing. A more detailed description is provided in the Supplementary Materials and Methods.

\section{Analysis of mRNA and miRNA data}

The raw CEL files were background-corrected, normalized, and summarized using frozen robust multiarray analysis (McCall et al. 2010). Background correction and quantile normalization were used to generate microarray expression values from all arrays passing quality controls and were performed using the custom chip definition files environment HGU133Plus2_Hs_ENTREZG v16.0 (Dai et al. 2005), as previously described in greater detail (Iskandar et al. 2018; Zanetti et al. 2018).

A model was fitted using limma software (Smyth 2004) to estimate the treatment effect (for each experimental factor combination item, concentration, and PE duration) by including the covariate exposure run as a blocking variable to account for the pairing during an exposure run (exposed vs. air-exposed control samples). The $p$ values for each computed effect were adjusted across genes using the Benjamini-Hochberg FDR method (Benjamini and Hochberg 1995). Differentially expressed genes were defined as a set of genes, whose FDR was $<0.05$.

\section{Causal network-based enrichment analysis of transcriptomic data}

Quantitative assessment of the transcriptomic data was conducted using a network enrichment approach and the network perturbation amplitude (NPA) algorithm (Martin et al. 2014). Briefly, the methodology aims to contextualize transcriptome profiles (treated vs. control samples) and quantify the biological impact of exposure by combining alterations in gene expression into differential network node values (i.e., 1 value for each node of a causal network model) (Boué et al. 2015). The causal network models themselves are built while considering the biology relevant for the tissue and context under the study (Boué et al. 2015). For example, the network models used here are those relevant to respiratory physiology (see Supplementary Table 2). This contextual information is typically not available from most existing knowledge bases (Khatri et al. 2012); therefore, their use would improve the specificity and sensitivity to infer the exposure impacts. The network models used for the analysis are given in Supplementary Table 2.

The NPA method uses transcriptome data without an FC or $p$ value cutoff. The differential node values were determined by fitting procedures inferring the values that best satisfy the directionality of the causal relationships contained in the network model (e.g., positive or negative signs). NPA scores carried a confidence interval accounting for experimental variation, and the associated $p$ values were computed. In addition, companion statistics were derived to permute the network structure, and the gene expression profiles were derived to inform the specificity of the NPA score to the biology reflected in the network models. The results from those permutation statistics were reported as $* \mathrm{O}$ and $\mathrm{K}^{*}$ if their $p$ values fell below the threshold of significance (0.05). A network was considered significantly perturbed by exposure if the 3 values (the $p$ value for experimental variation, ${ }^{*} \mathrm{O}$, and $\mathrm{K}^{*}$ ) were below 0.05 (Martin et al. 2014).

A system-wide metric for biological impact, the Biological Impact Factor (BIF) (Hoeng et al. 2012; Thomson et al. 2013) summarized the impacts of the exposure on the cellular system into a single (absolute) number, thus enabling a simple and high-level evaluation of the treatment effects across multiple timepoints. Calculating the BIF required the collection of all applicable network models (Supplementary Table 2) and involved aggregating the NPA score of each network.

\section{Statistical analyses and data repository}

Basic descriptive statistical measures, such as mean, median, and standard deviation (SD), for all of the investigated endpoints were computed (except for the analysis of mRNA and miRNA, see "Analysis of mRNA and miRNA data" section; and for the analysis of targeted proteomics, see "Target proteomics by PRM" section). 3R4F CS-, MESH Classic Tobacco aerosol-, or Base aerosol-exposed groups were compared with the air-exposed controls using paired $t$ test (paired within the same exposure chamber; the statistical comparisons focused on the differences between 3R4F CS-, MESH Classic Tobacco aerosol-, or Base aerosolexposed groups vs. their own corresponding air-exposed controls that were exposed in the same exposure chamber). 
The analyses were performed in R-3.2.2 or R-3.1.2. Data set, further detail on the protocols, and additional data visualizations are available on the INTERVALS platform at https://doi.org/10.26126/intervals.ri2tah.1 [2D NHBE and 3D small airway studies] and https://doi.org/10.26126/inter vals.kv8zst.1 [3D buccal study]). The mRNA array datasets can be accessed in the Arrays Express repository (ID: E-MTAB-7911). The miRNA array datasets can be accessed in the Arrays Express repository (ID: E-MTAB-7912).

\section{Results}

\section{First-layer assessment: effects of MESH Classic Tobacco and Base liquids, compared with those of 3R4F CS TPM, on cell viability of 2D NHBE cells}

The first layer of the proposed framework aims to screen e-liquids for their potential toxicity. In this context, we evaluated the impact of MESH Classic Tobacco liquid and Base e-liquids compared with that of 3R4F CS TPM on cell viability.

The viability of 2D NHBE cells was measured following a 24-h incubation with MESH Classic Tobacco e-liquid and its Base e-liquid, as well as 3R4F CS TPM, at different concentrations, using RTCA. RTCA measures electron flow transmitted between microelectrodes covering the bottom area of each well, where submerged cultures are seeded. Adhering cells disrupt the interaction between the electrodes, thus impeding the electron flow. This impedance-resistance to the altering electrical current-thus corresponds to the cell number, cell morphology/size, and the strength of the cell-cell attachment onto the plate (Yan et al. 2018).

Figure 2a shows a dose-dependent decrease in cell viability following a 24-h incubation with MESH Classic Tobacco e-liquid and its Base liquid (with a half maximal effective concentration $\left[\mathrm{EC}_{50}\right.$ ] of $476.7 \mu \mathrm{g}$ nicotine $/ \mathrm{mL}$ for $M E S H$ Classic Tobacco and $423.6 \mu \mathrm{g}$ nicotine/mL for Base e-liquid). The results showed that the effects on cell viability were similar between the 2 e-liquids with reference to their nicotine concentrations. Furthermore, decreased cell viability was detected following a 24-h incubation with 3R4F CS TPM at approximately 20-fold lower nicotine concentrations (with an $\mathrm{EC}_{50}$ of $19.3 \mu \mathrm{g}$ nicotine $/ \mathrm{mL}$ ).

\section{Second-layer assessment: effects of MESH Classic Tobacco and Base liquids, compared with those of 3R4F CS TPM, determined using HCS assays and 2D NHBE cells}

The second layer of the proposed framework aims to identify the toxicity-related mechanisms beyond the impact on cell viability. For this, we utilized HCS assays that rely on the use of simultaneous recording of multiple fluorescence readouts coupled with image processing and visualization tools to extract quantitative data (Xia and Wong 2012). A specific endpoint is detected using a specifically designed fluorogenic antibody/dye.

We incubated 2D NHBE cells for 4 and $24 \mathrm{~h}$ with the MESH Classic Tobacco e-liquid and its Base e-liquid, as well as with 3R4F CS TPM at different concentrations. Figure $2 \mathrm{~b}$ shows that a 4-h incubation with $3 \mathrm{R} 4 \mathrm{~F}$ CS TPM resulted in dose-dependent impact on DNA damage, glutathione content, oxidative stress, and stress kinase (c-Jun). More pronounced changes were seen for 3R4F CS TPM when the cells were incubated for $24 \mathrm{~h}$. Changes of these markers after incubation with MESH Classic Tobacco e-liquid or Base e-liquid were also detected, but at much higher nicotine concentrations, and the duration of the incubation did not largely influence the culture's response.

Moreover, to facilitate a better comparison across the tested items, we derived the MEC for each tested endpoint and for each tested item. The MEC indicates the dose of the test item at which we detected a response above the noise level (see Materials and Methods). Figure 2c shows the MECs at which 3R4F CS TPM, MESH Classic Tobacco e-liquid, and Base e-liquid elicited a response in 2D NHBE cells after they were incubated for 4 and $24 \mathrm{~h}$. The numbers at the outer pie chart correspond to the nicotine concentrations associated with the MECs; if the response (for all doses tested) was constant, we did not derive the MEC. The lower the MEC, the longer the arc length of 1 pie slice (i.e., $1 \mathrm{HCS}$ assay endpoint). Therefore, the arc length of the pie slice indicates the effect (potential toxicity) of each item tested. The results show that the arc lengths associated with $3 \mathrm{R} 4 \mathrm{~F}$ CS TPM were greater than those associated with MESH Classic Tobacco e-liquid and its Base e-liquid. Impacts in cell membrane permeability and cytochrome $\mathrm{c}$ release were not detected following the 4-h incubation with 3R4F CS TPM, but were detected following the 24-h incubation; this reflects the dose-response curves in Fig. 2b. The impacts of MESH Classic Tobacco and Base e-liquids were similar, with shorter arc length than those of the 3R4F CS TPM.

We list the calculated MEC - which is the minimum concentration at which we could detect effects-in Table 4. The results indicated that to reach an effect elicited by a 24-h incubation with 3R4F CS TPM, we needed to incubate 2D NHBE cells in MESH Classic Tobacco e-liquid at a concentration of $\sim 51$ times greater than the concentration of $3 \mathrm{R} 4 \mathrm{~F}$ CS TPM (622.83 divided by 12.09; see Table 4). Similarly, a 52-fold higher concentration of Base e-liquid was needed to trigger cellular responses that were detected following the 24-h incubation with 3R4F CS TPM (630.17 divided by 12.09; see Table 4). 


\section{A Cytotoxicity Measurement Using RTCA}

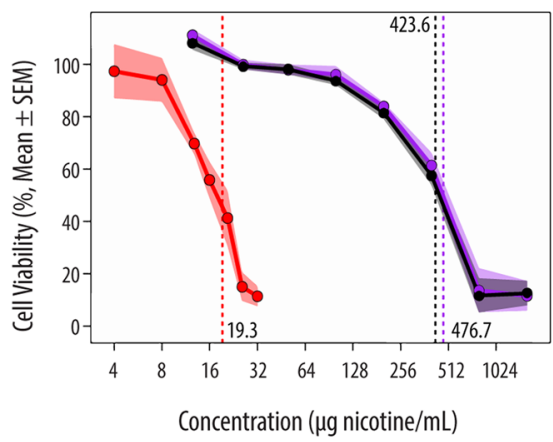

- 3R4F TPM

- MESH Classic Tobacco e-Liquid

- Base e-Liquid

\section{Minimum Effective Concentrations (MEC): Comparison Based on the High Content Screening Assays}
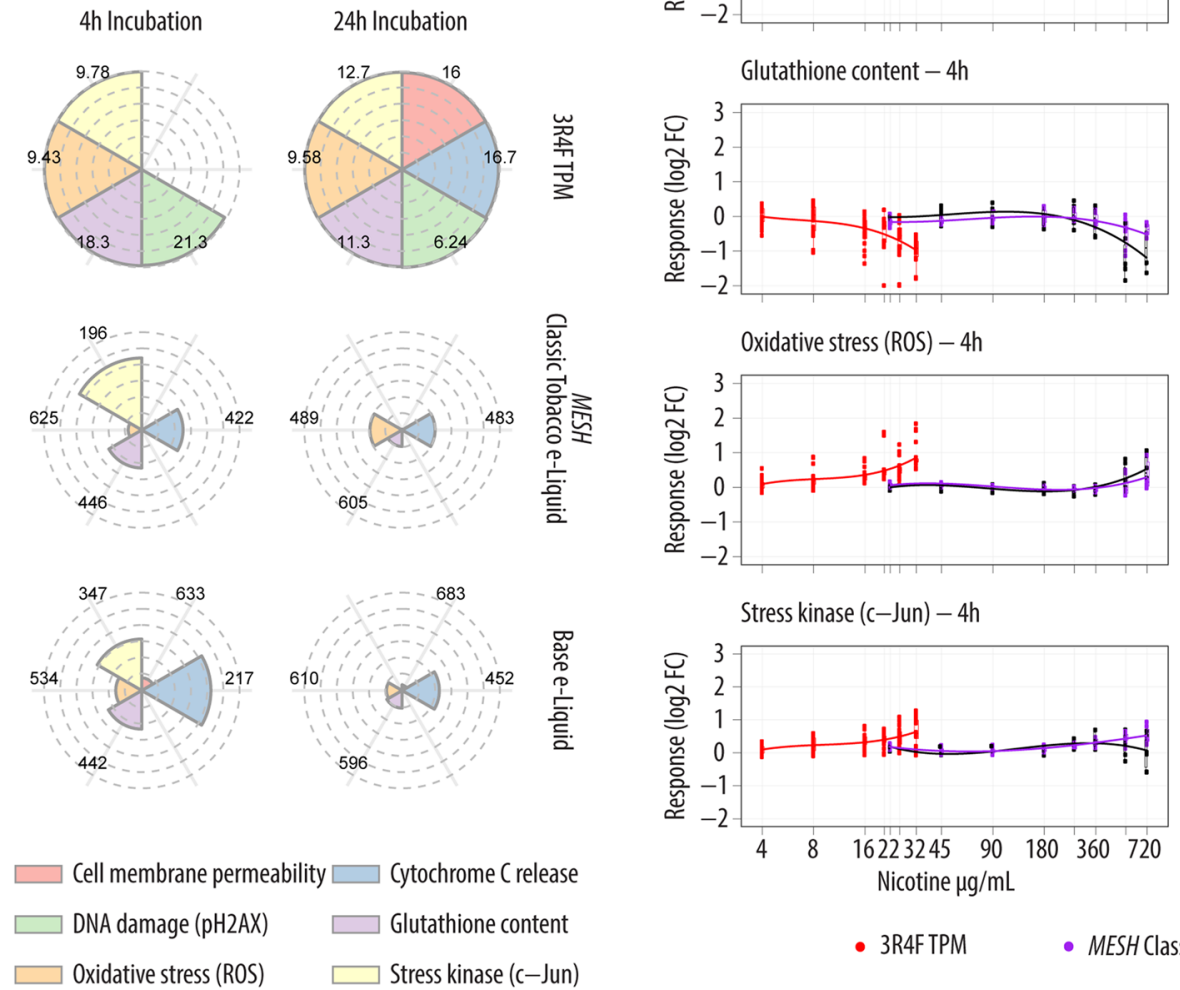

Cell membrane permeability $-24 \mathrm{~h}$

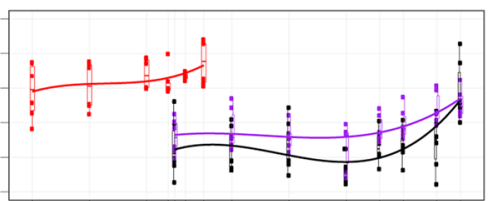

Cytochrome C release $-24 \mathrm{~h}$

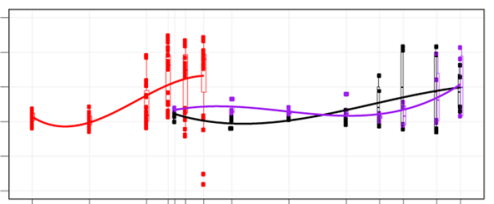

DNA damage ( $\mathrm{pH} 2 \mathrm{AX})-24 \mathrm{~h}$

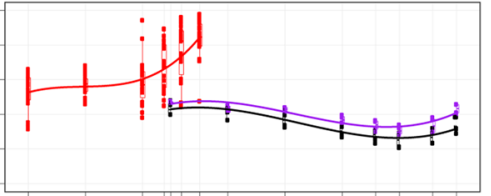

Glutathione content - 24h

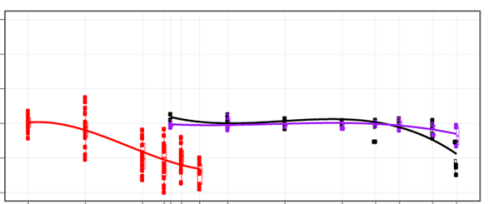

Oxidative stress (ROS) $-24 \mathrm{~h}$

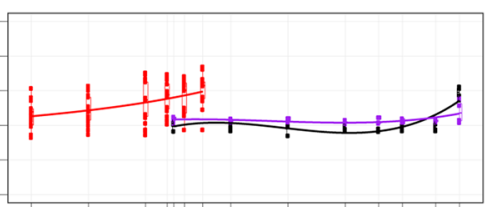

Stress kinase (c-Jun) - 24h

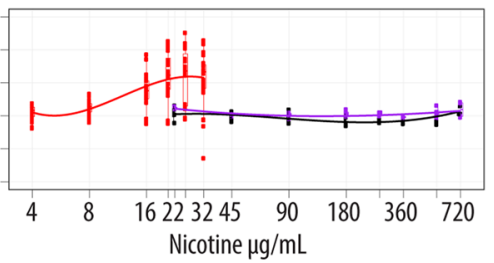

- 3R4FTPM - MESH Classic Tobacco e-Liquid - Base e-Liquid
Fig. 2 Cell viability in 2D NHBE cells and mechanistic investigation by HCS. a Cell viability in 2D NHBE cells incubated for $24 \mathrm{~h}$ with 3R4F CS TPM, MESH Classic Tobacco e-liquid, and Base e-liquid. Values are shown as the mean \pm standard error of the mean (SEM). b Output of HCS endpoints from 2D NHBE cells incubated with 3R4F CS TPM, MESH Classic Tobacco e-liquid, and Base e-liquid for 4 and $24 \mathrm{~h}(\boldsymbol{N}=3-8$ independent experiments). For each HCS assay, the
FCs of the response refer to the ratio of the signal detected following incubation with 3R4F CS TPM, MESH Classic Tobacco e-liquid, and Base e-liquid relative to the signal detected following incubation with the culture medium only. c Average MEC (from replicates of the independent experiments) for 3R4F CS TPM, MESH Classic Tobacco e-liquid, and Base e-liquid for each of the tested assays 
Table 4 Comparison of MECs

\begin{tabular}{|c|c|c|c|c|c|c|}
\hline \multirow[t]{2}{*}{ Toxicity measures (HCS assay endpoints) } & \multicolumn{3}{|l|}{$4 \mathrm{~h}$ incubation } & \multicolumn{3}{|l|}{$24 \mathrm{~h}$ incubation } \\
\hline & 3R4F CS TPM & $\begin{array}{l}M E S H \text { clas- } \\
\text { sic tobacco } \\
\text { e-liquid }\end{array}$ & Base e-liquid & 3R4F CS TPM & $\begin{array}{l}M E S H \text { clas- } \\
\text { sic tobacco } \\
\text { e-liquid }\end{array}$ & Base e-liquid \\
\hline Cell membrane permeability & $32^{\mathrm{a}}$ & $720^{\mathrm{a}}$ & 633 & 16 & $720^{\mathrm{a}}$ & 683 \\
\hline Cytochrome $\mathrm{C}$ release & $32^{\mathrm{a}}$ & 422 & 217 & 16.7 & 483 & 452 \\
\hline DNA damage (pH2AX) & 21.3 & $720^{\mathrm{a}}$ & $720^{\mathrm{a}}$ & 6.24 & $720^{\mathrm{a}}$ & $720^{\mathrm{a}}$ \\
\hline Glutathione content & 18.3 & 446 & 442 & 11.3 & 605 & 596 \\
\hline Oxidative stress (ROS) & 9.43 & 625 & 534 & 9.58 & 489 & 610 \\
\hline Stress kinase (c-Jun) & 9.78 & 196 & 347 & 12.7 & $720^{\mathrm{a}}$ & $720^{\mathrm{a}}$ \\
\hline $\begin{array}{l}\text { Mean MECs from all of the above toxicity } \\
\text { measures }\end{array}$ & 20.47 & 521.50 & 482.17 & 12.09 & 622.83 & 630.17 \\
\hline
\end{tabular}

${ }^{a}$ The MEC (given in $\mu \mathrm{g}$ nicotine/mL medium) was not derived, because the response (for all the tested doses) was constant. Thus, the number in the table refers to the highest tested concentrations

\section{Third-layer assessment: biological impacts of exposure to the whole aerosol of MESH Classic Tobacco compared with those of 3R4F CS using 3D ALI human buccal and small airway epithelial models}

The third layer of the proposed framework focuses on the investigation of the toxicity-related mechanism of EC aerosols, not e-liquids. For this, we conducted exposure experiments using ALI human organotypic buccal and small airway epithelial culture models. To fairly compare the effect of exposures (among MESH Classic Tobacco aerosol, Base aerosol, and 3R4F CS), the doses of MESH Classic Tobacco aerosol and Base aerosol applied to the cultures are those that resulted in at least similar or higher nicotine deposition (in the exposure chamber) than 3R4F CS (see Materials and Methods, Table 3).

Cytotoxicity was first evaluated by quantifying the activity of adenylate kinase released into the basolateral media of buccal (Fig. 3a) and small airway (Fig. 3b) cultures $48 \mathrm{~h}$ following exposure. For both culture types, we found that cytotoxicity in the cultures following exposure to the undiluted MESH Classic Tobacco or Base aerosols at the puff number tested were comparable with those exposed to air. In contrast, cytotoxicity levels in cultures exposed to the diluted 3R4F CS, at doses resulting in a lower deposited nicotine concentration than MESH Classic Tobacco or Base aerosols, were around $30 \%$ cytotoxicity.

Pronounced histological alterations were not seen in either buccal or small airway cultures $48 \mathrm{~h}$ following exposure to the undiluted MESH Classic Tobacco or Base aerosols, which resulted in nicotine concentrations up to around $159 \mu \mathrm{g} / \mathrm{mL}$ (for buccal) and $54 \mu \mathrm{g} / \mathrm{mL}$ (for small airway) (Fig. 4a, b). The morphology of the culture sections was similar to that of the air-exposed controls. Differently, buccal cultures that were exposed to $69 \%$ 3R $4 \mathrm{~F}$ CS for 112 puffs, which resulted in a deposited nicotine of around $92 \mu \mathrm{g} / \mathrm{mL}$, had marked cell alterations, apoptosis, dyskeratosis, and increased superficial desquamation. In small airway cultures, we detected marked increases in apoptosis and epithelial atrophy following exposure to only 13\% 3R4F CS for 112 puffs, which resulted in a deposited nicotine of only around $10 \mu \mathrm{g} / \mathrm{mL}$.

To investigate the molecular impact of exposure to MESH Classic Tobacco aerosol, we performed a mechanistic assessment using 2 approaches: (1) a causal network enrichment analysis based on the transcriptome profiles and (2) a targeted protein analysis of the secreted inflammatory mediator and selected markers in epithelial cells. For this mechanistic investigation, we excluded samples that already exhibited marked damage (i.e., the buccal cultures exposed to $69 \%$ 3R 4F CS for 112 puffs and small airway cultures exposed to 13\% 3R4F CS for 112 puffs, see Fig. 4a, b). This is because cellular and molecular changes in an already damaged tissue would merely reflect these morphological alterations (Davis et al. 2013; Janovitz and Wallig 2013). Furthermore, for comparison purposes, we focused on examining the cultures that were exposed to the undiluted MESH Classic Tobacco or Base aerosols for the same puff number (i.e., 112 puffs).

The first approach for the mechanistic investigation, the causal network enrichment analysis, uses transcriptome data and the $p$ value threshold-free NPA algorithm (Martin et al. 2014). The NPA method computes exposure-induced perturbation scores of various biological processes using the sample transcriptome profiles to contextualize the exposure effect by deriving the alterations in transcriptomes into differential node values of causal networks (Fig. 5a); a more detailed description of the approach was reported previously (Hoeng et al. 2014). The network models can be further 


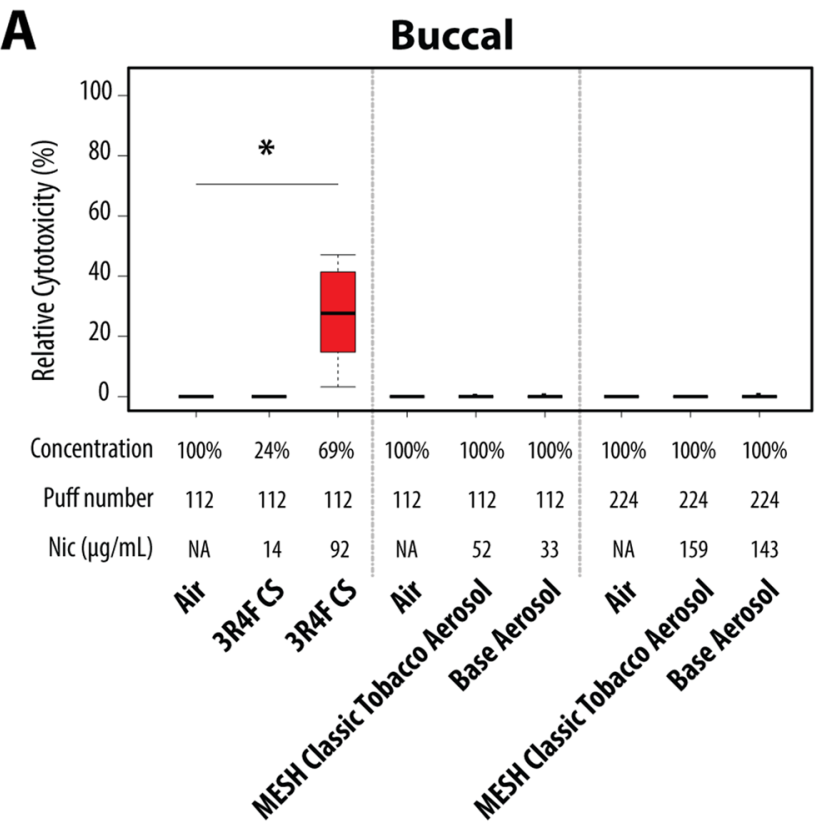

Fig. 3 Cytotoxicity in human buccal and small airway epithelial cultures following exposure. Relative cytotoxicity is shown as mean \pm SEM ( $N=9$ per group) for a buccal and $\mathbf{b}$ small airway cultures ( ${ }^{*} p$ value $\leq 0.05$ compared with air-exposed controls). The relative cytotoxicity was determined based on the release of adenylate

summarized into 4 main cellular processes (Cell Fate, Cell Proliferation, Cell Stress, and Inflammatory Process Network families), and ultimately, an overall perturbation score, which is termed the BIF, can be computed.

In this study, we generated the transcriptome data from the buccal and small airway epithelial cultures collected 4 , 24 , and $48 \mathrm{~h}$ following exposure. From the transcriptome data, we derived the perturbation of each network model analyzed (the perturbations of each network are given in Supplementary Fig. 2). Figure 5b, c shows that at any PE timepoint analyzed, perturbations of Cell Fate, Cell Proliferation, Cell Stress, and Inflammatory Process Network families following exposure to undiluted MESH Classic Tobacco or Base aerosols for 112 puffs were generally lower than the perturbation following 3R4F CS. An exception was seen in buccal cultures for Inflammatory Process Network family at the 4 and $24 \mathrm{~h}$ PE timepoints, i.e., the perturbation following Base aerosol was greater than following 3R4F CS (Fig. 5b). Note that the concentrations of deposited nicotine following MESH Classic Tobacco or Base aerosols were higher than those following 3R4F CS.

We further calculated the overall impact-a term we refer to as the "BIF"-in buccal (Fig. 5d) and small airway (Fig. 5e) cultures. The highest BIF score was taken as $100 \%$ and assigned to the reference group (termed "REF" in Fig. 5d, e). At any given timepoint analyzed, the highest BIF score for buccal cultures was seen for 3R4F CS, followed
B

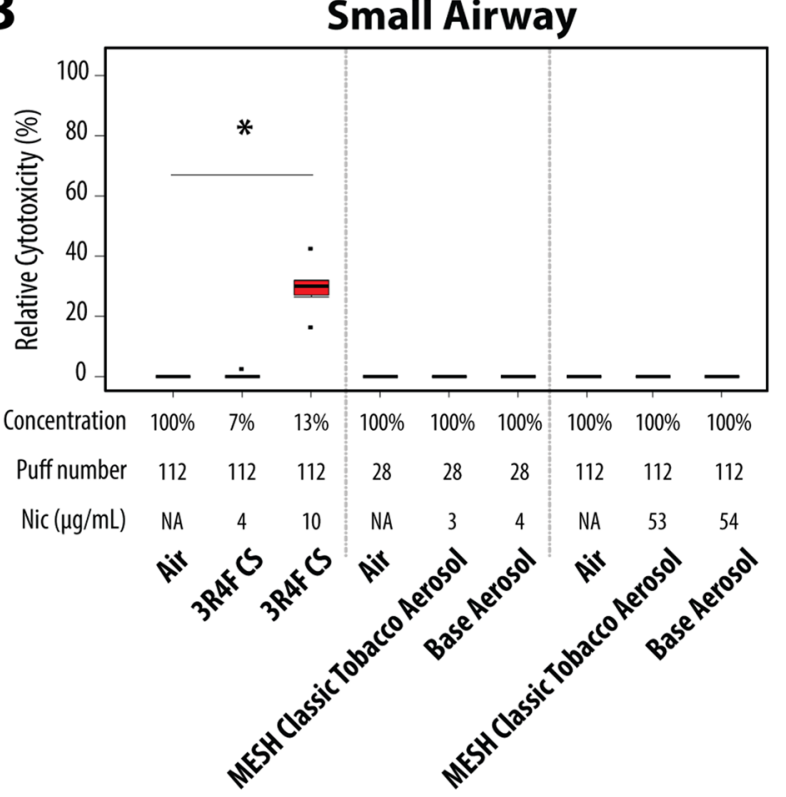

kinase from the epithelial cultures to the basolateral media at $48 \mathrm{~h} \mathrm{PE}$ (Triton X-treated cultures were considered to have $100 \%$ cytotoxicity, and non-exposed [incubator control] cultures were considered to have $0 \%$ cytotoxicity). NA not applicable, Nic nicotine

by Base aerosol and finally MESH Classic Tobacco aerosol. For small airway cultures, the highest BIF score was also detected for 3R4F CS, followed by similar BIF values (lower than the BIF for 3R4F CS) for both MESH Classic Tobacco and Base aerosols. The BIF scores in buccal cultures also show that the impact of exposure to MESH Classic Tobacco or Base aerosols dissipated after $48 \mathrm{~h}$. However, in small airway cultures, the impact had already diminished after $24 \mathrm{~h}$.

Interestingly, analyses of the global changes in the miRNAs showed that none of the miRNAs were significantly altered following exposure to MESH Classic Tobacco or Base aerosols in both buccal and small airway cultures (with the exception of 1 miRNA-miR-320b-that was found differentially increased only at the $24 \mathrm{~h}$ PE timepoint following Base aerosol exposure in small airway cultures). In contrast, significant alterations in many miRNAs were detected following exposure to 3R4F CS (Supplementary Fig. 3).

For the second approach of the mechanistic investigation, we quantified markers of proteins in the epithelial culture samples using PRM, a targeted mass spectrometry (MS)-based approach. The evaluated markers included proteins that are linked to relevant processes, such as xenobiotic metabolism response, oxidative stress, and autophagy. In both buccal and small airway epithelial cultures, exposure to the undiluted MESH Classic Tobacco or Base aerosols for 112 puffs was not linked to any marked alterations in the abundance of these proteins. Exposure 


\section{A Hematoxylin and Eosin-stained Sections (Buccal Epithelial Cultures)}

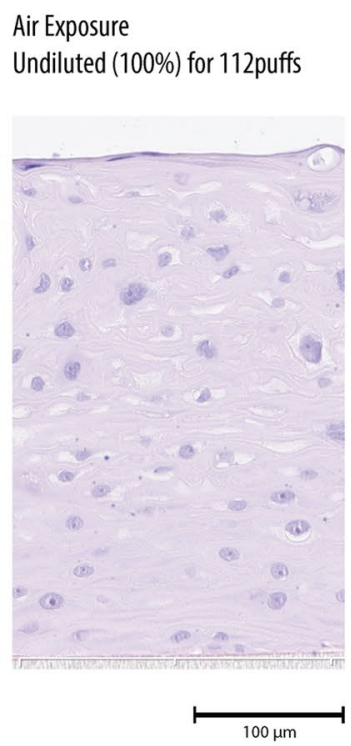

$3 R 4 F C S$

Diluted (24\%) for 112 puffs

$14 \mu \mathrm{g}$ nicotine $/ \mathrm{mL}$

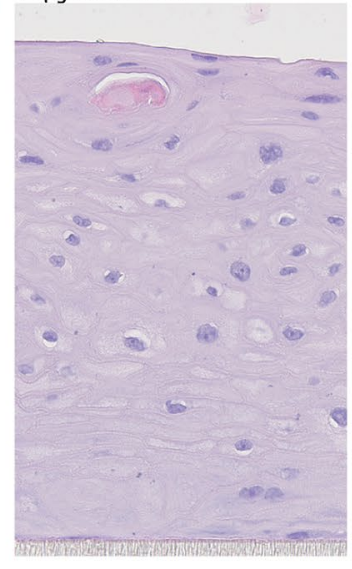

3R4F CS

Diluted (69\%) for 112 puffs

$92 \mu \mathrm{g}$ nicotine $/ \mathrm{mL}$

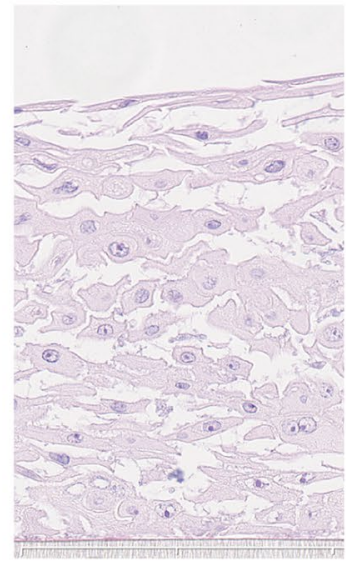

MESH Classic Tobacco Aerosol Undiluted (100\%) for 112 puffs

$52 \mu \mathrm{g}$ nicotine/mL

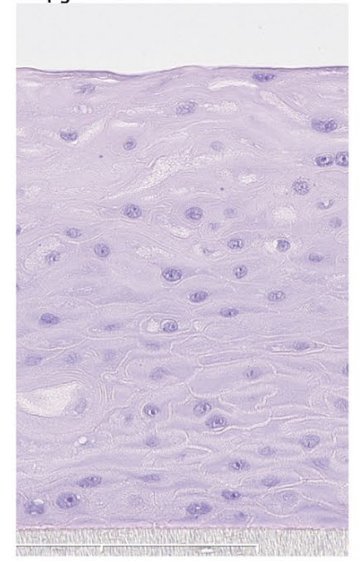

MESH Classic Tobacco Aerosol

Undiluted (100\%) for 224 puffs

$159 \mu \mathrm{g}$ nicotine/mL

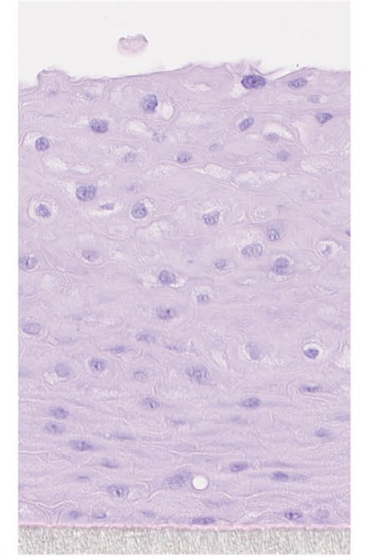

Base Aerosol

Undiluted (100\%) for 112 puffs

$33 \mu \mathrm{g}$ nicotine $/ \mathrm{mL}$

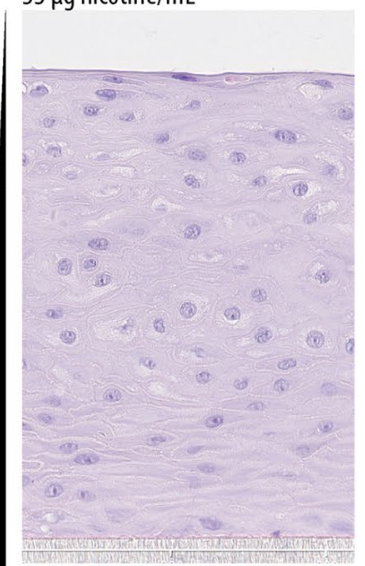

Base Aerosol

Undiluted (100\%) for 224 puffs

$143 \mu \mathrm{g}$ nicotine/mL

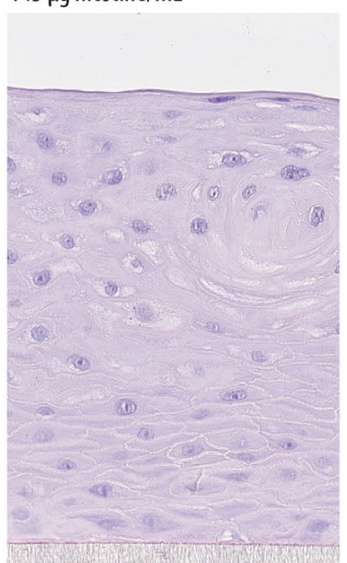

\section{B Hematoxylin, Eosin- and Alcian Blue-stained Sections (Small Airway Epithelial Cultures)}

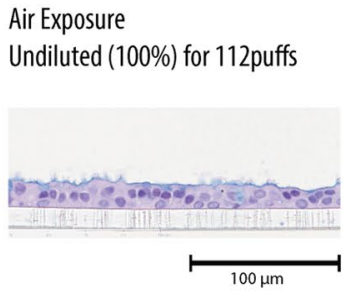

Air Exposure

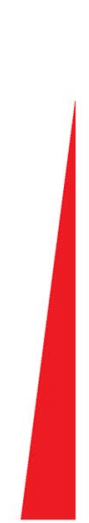

3R4F CS

Diluted (7\%) for 112 puffs $4 \mu \mathrm{g}$ nicotine $/ \mathrm{mL}$

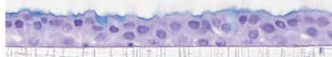

3R4F CS

Diluted (13\%) for 112 puffs $10 \mu \mathrm{g}$ nicotine $/ \mathrm{mL}$

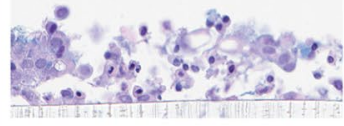

MESH Classic Tobacco Aerosol Undiluted (100\%) for 28 puffs $3 \mu \mathrm{g}$ nicotine/mL

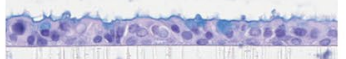

MESH Classic Tobacco Aerosol Undiluted (100\%) for 112 puffs $53 \mu \mathrm{g}$ nicotine $/ \mathrm{mL}$

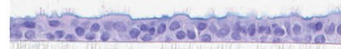

Base Aerosol Undiluted (100\%) for 28 puffs $4 \mu \mathrm{g}$ nicotine $/ \mathrm{mL}$

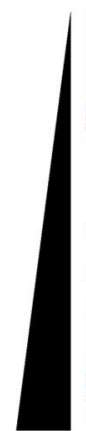

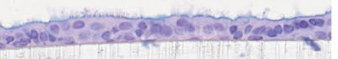

Base Aerosol

Undiluted (100\%) for 112 puffs

$54 \mu \mathrm{g}$ nicotine $/ \mathrm{mL}$

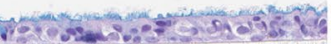

Fig. 4 Histology of human buccal and small airway epithelial cultures. a Representative images of the hematoxylin and eosin-stained buccal culture sections and $\mathbf{b}$ hematoxylin, eosin, and Alcian bluestained small airway culture sections are shown. The cultures were collected $48 \mathrm{~h}$ following exposure to the air, 3R4F CS, MESH Classic Tobacco aerosol, or Base aerosol for various doses as indicated. The concentrations of nicotine deposited in PBS in the exposure chamber are indicated in $\mu \mathrm{g} / \mathrm{mL}$ 
to the already diluted 3R4F CS for 112 puffs was linked to significant increase in the abundance of cytochrome P450 (CYP) 1A1 and CYP1B1 proteins (around 60-fold) relative to the abundance of the proteins following air exposure (Fig. 6a). ALDH1A3 protein abundance was also increased in cultures exposed to 3R4F CS exposure, although to a lesser degree than CYP enzymes. In buccal cultures, GCLC and NQO1 (oxidative stress markers), SQSTM1 (autophagy marker), and ALDH3A1 protein abundances were also altered following exposure to $3 \mathrm{R} 4 \mathrm{~F}$ $\mathrm{CS}$. We measured other protein markers; however, they were not significantly altered following any of the exposure conditions tested (Supplementary Fig. 4).

Furthermore, because the network analysis suggested that inflammatory response was elicited following exposure to MESH Classic Tobacco aerosol, we gathered additional information by analyzing the profile of secreted inflammatory mediators from the epithelial cultures using MAP analysis. We collected the basolateral media $48 \mathrm{~h}$ $\mathrm{PE}$ and measured the concentrations of various mediators involved in inflammatory response.

Figure $6 \mathrm{~b}$ shows the concentrations of the mediators that were altered $48 \mathrm{~h}$ following exposure to MESH Classic Tobacco aerosol, Base aerosol, 3R4F CS, or air. In both buccal and small airway cultures, increased matrix metalloproteinase (MMP)-1 and decreased chemokine (C-X-C motif) ligand (CXCL) 10 (also known as IP-10) concentrations were detected following exposure to 3R4F CS. In contrast, their concentrations were not altered in the basolateral media of cultures exposed to MESH Classic Tobacco or Base aerosols. For other mediators, they were altered depending on the exposure and culture types. In buccal cultures, decreased concentrations of CXCL 1 (also known as GRO $\alpha$ ) and increased concentrations of interleukin (IL)-1 $\beta$ were detected following 3R4F CS exposure; however, they were not significantly altered following exposure to MESH Classic Tobacco or Base aerosols. In turn, IL- $1 \alpha$ concentrations were found to be greater in the media of buccal cultures exposed to MESH Classic Tobacco or Base aerosols, but only slightly increased in the media of cultures exposed to 3R4F CS. In small airway cultures, increased vascular endothelial growth factor A (VEGFA), IL-8, and tissue inhibitor of metalloproteinase (TIMP)-1 secretion levels were found in cultures exposed to $3 \mathrm{R} 4 \mathrm{~F} \mathrm{CS}$, but not in the media of cultures exposed to MESH Classic Tobacco or Base aerosols.

Other mediators (e.g., IL- $1 \alpha$ and IL- $1 \beta$ in small airway cultures and VEGFA, IL-8, and TIMP-1 in buccal cultures) were also measured; however, their levels, including in the air-exposed cultures, were around and/or below the limit of the quantification (LOQ) (data not shown).

\section{Discussion}

The challenges for testing the potential toxicity of EC aerosols come not only from the apparent increased availability of EC devices, but also from the numerous flavors in the e-liquids. A robust approach is thus needed to test the potential toxicity of the final EC liquids and, eventually, the aerosolized formulations. Previously, we proposed a three-layer system toxicology framework for testing e-liquids (Iskandar et al. 2016). It starts with assessing the effects of e-liquids on cell viability (first layer), followed by investigating the potential mechanisms of toxicity elicited by e-liquids (second layer) and finally assessing the impacts of aerosols (third layer) (Iskandar et al. 2016). In the present work, we showed how we leveraged the three-layer framework to evaluate the potential toxicity and biological effects of the MESH Classic Tobacco and Base e-liquids/aerosols compared with those of 3R4F CS.

The results from the first-layer assessment suggested that the addition of MESH Classic Tobacco flavor mix is unlikely to alter the cytotoxicity of its Base e-liquid. We found that the $\mathrm{EC}_{50}$ value of $2 \mathrm{D}$ NHBE cells following incubation with MESH Classic Tobacco was similar to those following incubation with Base e-liquids. They were approximately 23 -fold higher than those following incubation with 3R4F CS TPM with reference to their nicotine concentrations. Our observation, however, was different from that reported earlier (Stevenson et al. 2019), showing that the addition of flavors (of other brands) increased the cytotoxicity of the base e-liquids when measured using the human myeloid leukemia-derived cell line. We noted that in that study, the commercial e-liquids were not directly compared with each corresponding base e-liquid (i.e., the commercial formulations were merely tested along with other formulations that do not contain flavors, regardless of whether the concentrations of PG, VG, or nicotine were similar). In addition, the e-liquids tested and the cell model used in that study were also different from what we used here. These aspects could likely explain the discrepancy between the results reported here and those in the earlier study (Stevenson et al. 2019).

Furthermore, to investigate whether the increased cytotoxicity was influenced by hyperosmolarity, which was reported previously for PG and G (Chaumont et al. 2019; Gonzalez-Suarez et al. 2017), we measured the osmolarity of the e-liquids. We found that the increased cytotoxicity associated with MESH Classic Tobacco and Base e-liquids was directly correlated with the osmolarity (Supplementary Fig. 5). This finding suggested that osmolarity could be a confounding factor in toxicity measures for e-liquids, an observation we previously highlighted in the context of the multi-layer framework (Iskandar et al. 

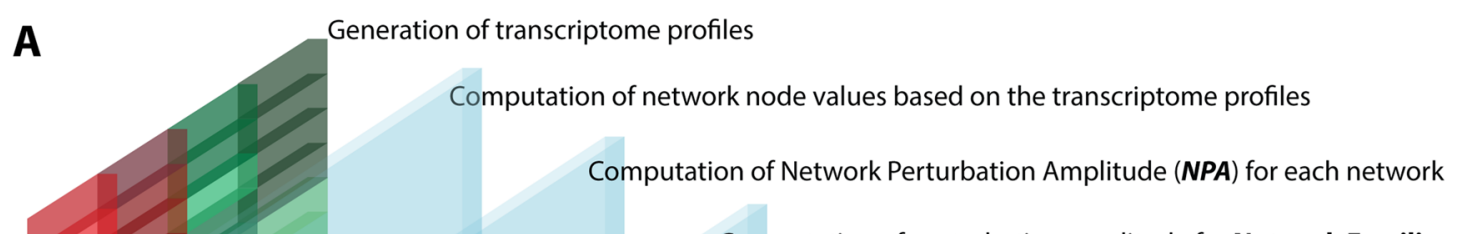

B

$4 \mathrm{~h}$ PE
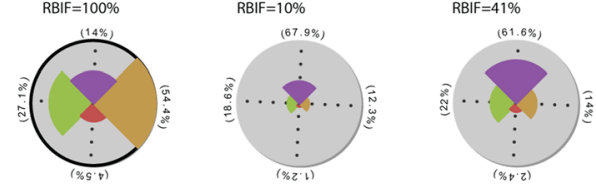

$24 \mathrm{hPE}$
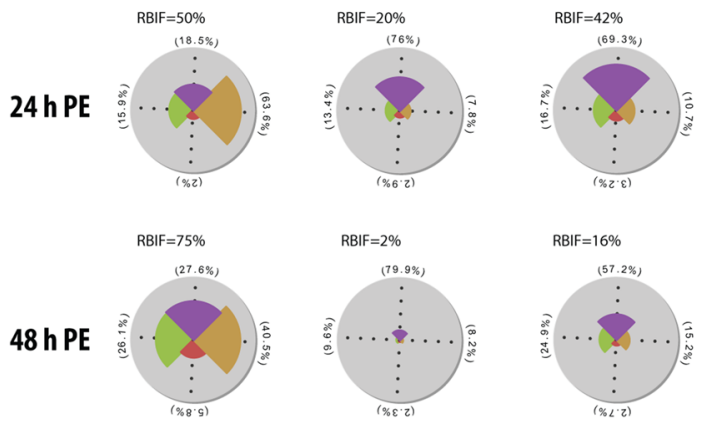

D

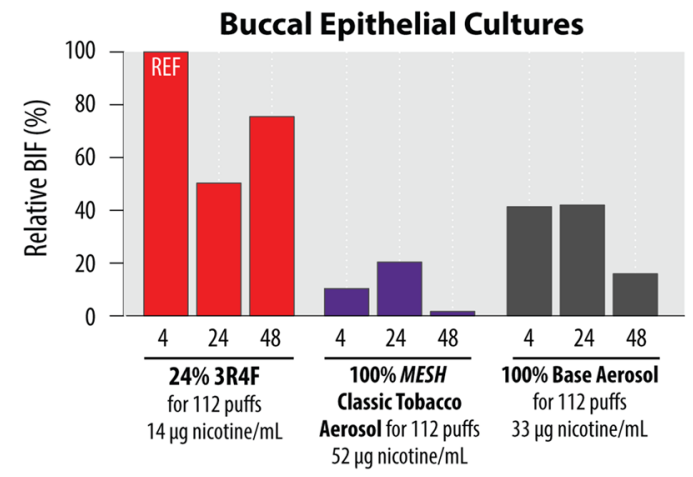

Computation of perturbation amplitude for Network Families

Computation of the overall perturbation

(BIF, biological impact factor)

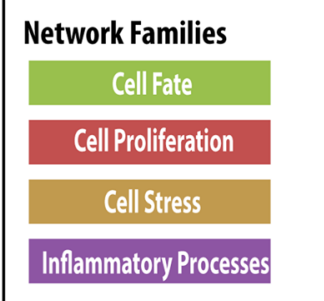

C

Small Airway Epithelial Cultures

$4 \mathrm{hPE}$

$\begin{array}{ccc}7 \% 3 \text { R4F CS } & 100 \% \text { MESH Classic Tobacco } & 100 \% \text { Base Aerosol } \\ \text { for } 112 \text { puffs } & \text { Aerosol for } 112 \text { puffs } & \text { for } 112 \text { puffs } \\ 4 \mu \mathrm{g} \text { nicotine } / \mathrm{mL} & 53 \mu \mathrm{g} \text { nicotine } / \mathrm{mL} & 54 \mu \mathrm{g} \text { nicotine } / \mathrm{mL}\end{array}$

$24 \mathrm{hPE}$
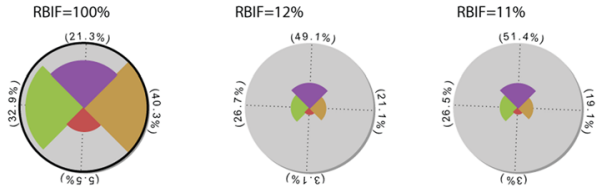

$48 \mathrm{~h}$ PE
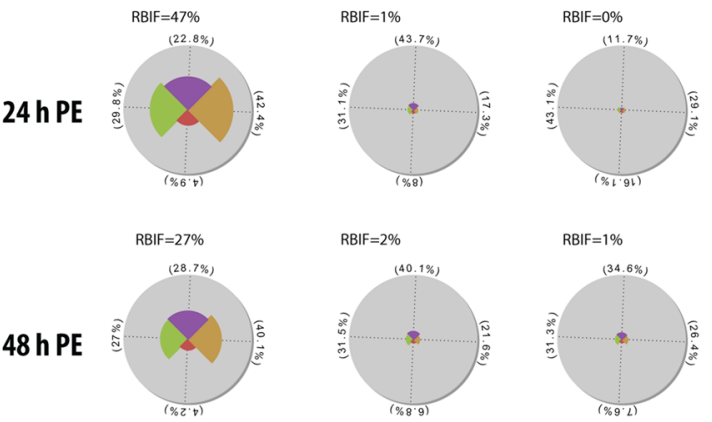

E

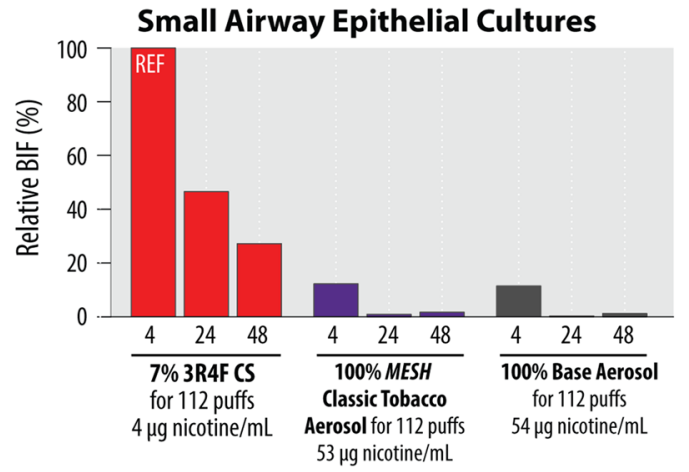


4Fig. 5 Causal network enrichment analysis on the transcriptome profiles. a From the global gene expression changes between exposed and air-exposed control samples, we used a collection of toxicological causal biological network models to deduce the biological impact of an exposure on a given network using the NPA algorithm (see Materials and Methods). Thus, the overall impact, termed BIF, can be deduced. The BIF takes into account the overall perturbations of all networks analyzed. Perturbations of the network families 4, 24, and $48 \mathrm{~h}$ following exposure to the 3R4F CS or EC aerosols for 112 puffs in $\mathbf{b}$ buccal and $\mathbf{c}$ small airway cultures are shown as pie charts. For a given culture type, the contrast with the highest perturbation is marked with a thick black line (taken as $100 \% \mathrm{BIF}$, or the reference "REF"). The overall BIF for the corresponding PE timepoints is shown in $\mathbf{d}$ and $\mathbf{e}$, respectively

2016). Osmolarity of the e-liquids should thus be evaluated in every study, where e-liquid toxicity is investigated.

The HCS assay results from the second-layer assessment were complimentary to those obtained from the first-layer assessment. No major differences in cell membrane permeability, cytochrome c release, DNA damage, GSH content, oxidative stress, and stress kinase were detected between cultures incubated with MESH Classic Tobacco e-liquid and with Base e-liquid. This observation supported the premise that the addition of flavors (in the MESH Classic Tobacco e-liquid) is unlikely to elicit molecular changes distinct from those elicited following treatment with the Base e-liquid only. The HCS assay results also showed that various biological processes were markedly impacted following incubation with 3R4F CS TPM, even though they were administered to the cultures at much lower nicotine concentrations than MESH Classic Tobacco e-liquid or its Base e-liquid (the highest concentrations of MESH Classic Tobacco or Base e-liquids tested contained 23 times higher nicotine than the highest tested concentration of 3R4F CS TPM). This finding suggested that the potential toxicity of MESH Classic Tobacco or Base e-liquids to 2D NHBE cells was much lower than the toxicity of 3R4F CS TPM when compared based on their nicotine levels.

Evaluating the e-liquids can still provide insights into their potential toxicity; however, we also recognize that in reality, the human respiratory system will be exposed to the aerosol and not to the e-liquid. Certain chemicals, despite being approved for ingestion, have been shown to elicit adverse effects when inhaled (e.g., diacetyl, acetylpropionyl, acetoin, cinnamaldehyde, and benzaldehyde) (NAS 2018). Although some may rely on the "Generally Recognized as Safe" (GRAS) status to justify the use of certain flavors in e-liquid formulations, the GRAS status only signifies that the flavors are safe for oral administration and use in food products, but does not prove that the flavors are safe for inhalation (NAS 2018). Therefore, in the third layer of the assessment framework, we tested the biological impacts of exposure to MESH Classic Tobacco and Base aerosols.
The aerosolization process might yield other products originated from the thermal decomposition of the flavors that occurs at high temperatures (Czégény et al. 2016; Wang et al. 2017). Therefore, we also measured the concentrations of various carbonyls that were deposited into PBS that was placed into the base module of the exposure chambers. From this experiment, following exposure to undiluted MESH Classic Tobacco or Base aerosols for 480 puffs, the concentrations of carbonyls-formaldehyde, acetaldehyde, acetone, acrolein, propionaldehyde, crotonaldehyde, methyl ethyl ketone, and butyraldehyde-were below the LOQ (Supplementary Table 3). This is in contrast to what can be detected following exposure to 3R4F CS: the levels of carbonyls reached to up to 40-fold the lower limit of quantification (LLOQ) (Supplementary Table 3).

The results of the third-layer assessment showed that exposure to MESH Classic Tobacco or Base aerosols, which resulted in concentrations of deposited nicotine up to $54 \mu \mathrm{g} /$ $\mathrm{mL}$, did not cause cytotoxicity and tissue damage to small airway cultures, while the diluted 3R4F CS, which resulted in a concentration of deposited nicotine of $10 \mu \mathrm{g} / \mathrm{mL}$, was cytotoxic and caused tissue damage in small airway cultures. In buccal epithelial cultures, MESH Classic Tobacco or Base aerosols at a dose resulting in concentrations of deposited nicotine up to $159 \mu \mathrm{g} / \mathrm{mL}$ were non-toxic, while the diluted 3R4F CS that resulted in a concentration of deposited nicotine around $92 \mu \mathrm{g} / \mathrm{mL}$ caused marked cytotoxicity and tissue damage. Compared with the concentrations of nicotine found in the saliva of EC users (Papaseit et al. 2017), these concentrations were already overestimations of the human exposure: Papaseit and colleagues reported that the maximum nicotine concentrations in the saliva of EC users after 2 consecutive sessions (with 10 puffs per session) were only around $0.86 \mu \mathrm{g} / \mathrm{mL}$ (Papaseit et al. 2017).

Based on the global gene expression profiles, among the 4 network families tested, exposure to the undiluted $M E S H$ Classic Tobacco or Base aerosols had a more pronounced impact mainly on the Inflammatory Process Network family. Nonetheless, at the $48 \mathrm{~h}$ post-exposure timepoint, the perturbations of Inflammatory Process Network family following exposure to undiluted MESH Classic Tobacco or Base aerosols were less than the perturbation following diluted 3R4F CS exposure in both buccal and small airway cultures. These results suggested that there was an early inflammatory response triggered following exposure to MESH Classic Tobacco or Base aerosols; however, the cultures could finally recover.

Interestingly, for buccal cultures, exposure to Base aerosol resulted in slightly higher overall perturbations (up to 40\% relative BIF) than exposure to MESH Classic Tobacco aerosol (up to $\sim 20 \%$ relative BIF). This slightly higher relative BIF scores of Base aerosol compared with MESH Classic Tobacco aerosol in the buccal cultures, however, was not 
A Alterations in Protein Abundance in the Epithelial Cultures

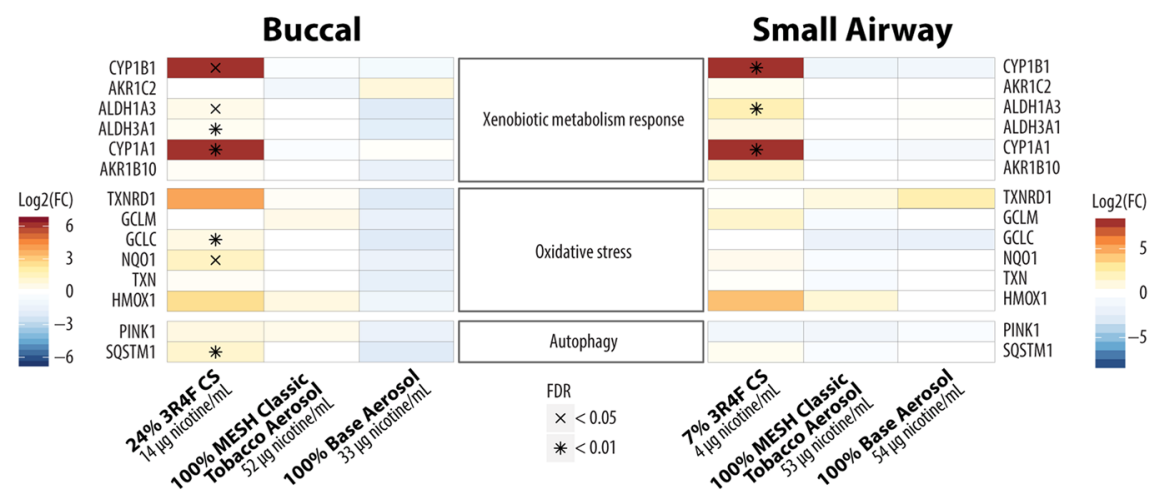

B Alterations in Inflammatory Mediator Proteins Secreted from the Epithelial Cultures
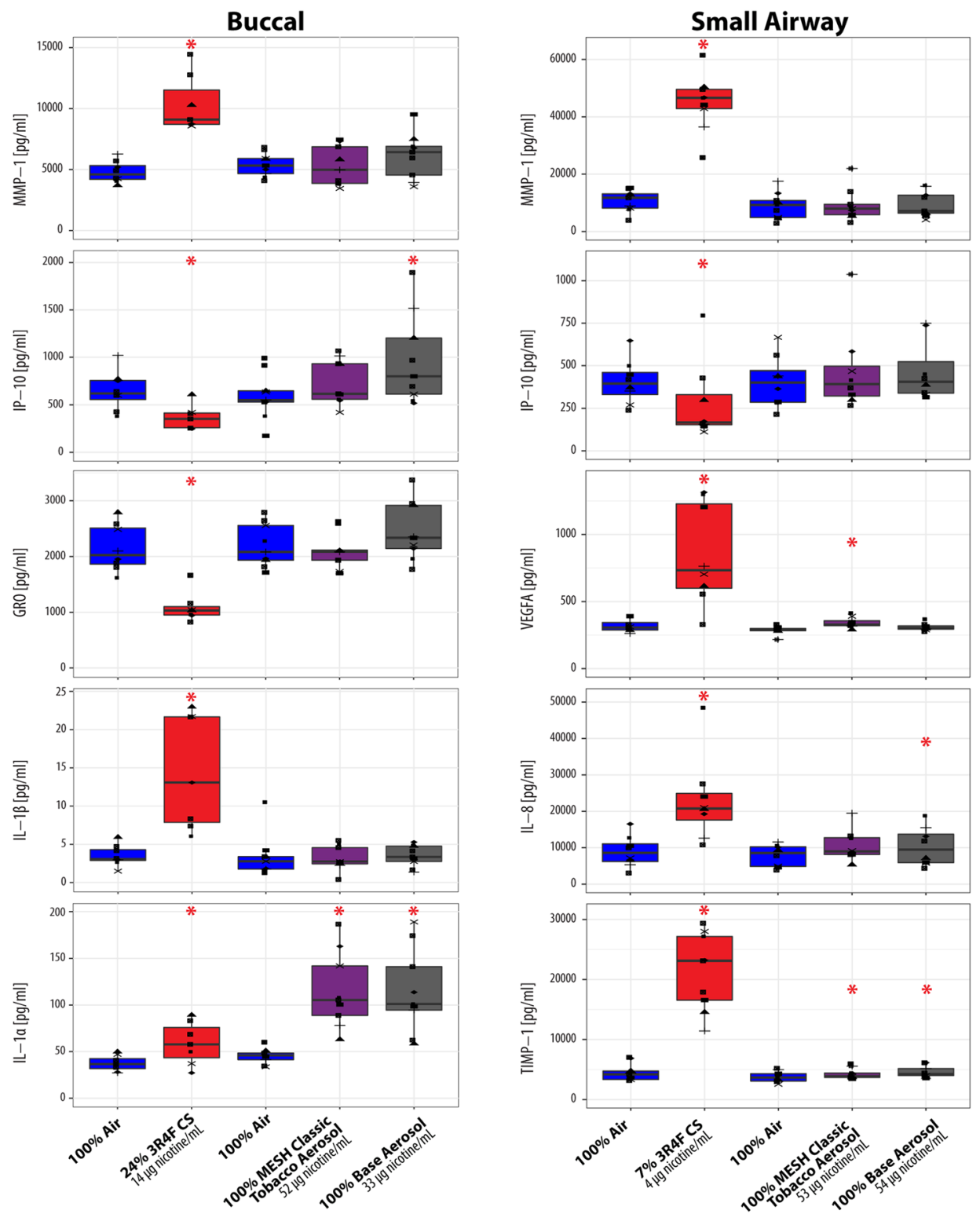
4Fig. 6 Alterations in protein markers following 3R4F CS and EC aerosol exposure. a Set of proteins was quantified using targeted MSbased proteomics for the buccal epithelial cultures collected $24 \mathrm{~h}$ following exposure and small airway epithelial cultures collected $48 \mathrm{~h}$ following exposure. Each row represents 1 quantified protein, and each column represents a comparison between an exposed sample and air-exposed controls. b Set of mediators regulating inflammatory response was quantified using Luminex-based multiplex assay from the basolateral medium of the cultures collected $48 \mathrm{~h}$ following exposure. Star symbol (*) indicates $p$ value $\leq 0.05$ compared with the airexposed samples

corroborated by the other endpoints measured in this study, such as cytotoxicity, histology, selected protein markers, concentrations of secreted mediators, and miRNA profile. This leads us to think that the slightly greater overall perturbations had little implication in the physiological processes of buccal cultures.

Such a tissue-specific response was also reflected from the profiles of secreted mediators that were altered following exposure. Tissue-specific soluble factors are important to condition the local environment against stimuli or pathogens (Hu and Pasare 2013). Decreased GRO and increased IL-1 $\beta$ secretion following 3R4F CS exposure were only detected in buccal cultures but not in small airway cultures, while marked increased secretion in VEGFA, IL-8, and TIMP-1 following 3R4F CS exposure was seen only in small airway cultures but not in buccal cultures; these observations were consistent with our previous finding (Iskandar et al. 2019). These mediators were not altered pronouncedly following exposure to MESH Classic Tobacco or Base aerosols.

Nonetheless, we were intrigued by the pronounced increase in IL- $1 \alpha$ secretion from buccal cultures following exposure to MESH Classic Tobacco or Base aerosols, despite the absence of tissue damage. This observation was also seen previously in buccal cultures following exposure to an EC aerosol of a prototype "Test Mix" and its base formulation (Iskandar et al. 2019). The expression of the ILIA gene, however, was not markedly impacted by MESH Classic Tobacco and Base aerosol exposure (data not shown). The absence of cytotoxicity and tissue damage in these cultures prompted us to assume that the increase IL- $1 \alpha$ secretion could be a stress response specific for stratified keratinized epithelium (i.e., buccal epithelium) triggered by a highly exaggerated exposure concentration (as discussed earlier, the EC aerosol nicotine concentrations tested were around 60 times higher than human saliva concentrations following EC use $[\sim 53 \mu \mathrm{g}$ nicotine $/ \mathrm{mL}$ in this in vitro study vs. $\sim 0.86 \mu \mathrm{g}$ nicotine/mL in human samples (Papaseit et al. 2017)]. This would be aligned with an earlier premise that IL-1 $\alpha$ secretion from cells that maintain their integrity may be a stresssensing mechanism demonstrating cellular stress resolution and recovery (Kim et al. 2013; Rider et al. 2017). IL-1 $\alpha$ is also found constitutively present in keratinocytes, epithelial cells, and endothelial cells under normal conditions, unlike IL-1 $\beta$, which mainly elevated under pathological conditions (Dinarello 2018). Future studies should aim for a more comprehensive assessment of the role of IL- $1 \alpha$ in buccal epithelium following exposure to EC.

One of the limitations of the study is attributed to the lack of data on submerged oral epithelial cells using the first- and second-layer assessment, even though we tested the impact of the smoke/aerosols on the 3D ALI buccal epithelial cultures. The correlation between data obtained using these two systems (submerged 2D vs. ALI 3D cultures) could be useful to estimate the potential toxicity of certain e-liquid formulations, as in an initial screening approach. For example, in the present study, the first-layer assessment showed that $3 \mathrm{R} 4 \mathrm{~F}$ CS TPM containing $10 \mu \mathrm{g}$ nicotine $/ \mathrm{mL}$ resulted in a $30 \%$ reduction in cytotoxicity of submerged primary bronchial epithelial cells (based on RTCA), while the thirdlayer assessment showed that 3R4F CS at a dose resulting in around $10 \mu \mathrm{g}$ deposited nicotine/well also resulted in around $30 \%$ cytotoxicity in ALI small airway cultures (based on AK assay). Therefore, this observation suggested that the information obtained in the first- and second-layer assessment may still provide a relevant indication to the potential toxicity of the aerosolized formulations.

A standardization in EC-testing approaches is lacking (Iskandar et al. 2016). For example, standardized puffing regimens for CS are available from various entities such as the Federal Trade Commission, International Organization for Standardization, Cooperation Centre for Scientific Research Relative to Tobacco (CORESTA), and Health Canada. In contrast, a standardized puffing regimen for ECs does not exist, except one that is proposed by CORESTA (i.e., the CORESTA Recommended Method No. 81, (CORESTA 2015)). Furthermore, a standardized battery of assays for in vitro toxicity testing of ECs and a standard reference EC are also not available. These issues have challenged a proper comparison across studies conducted in different laboratories. A joined force across sectors (regulatory authorities, industry, and academia) is needed to collaboratively define a robust study design with appropriate sample size and statistical analysis that meets the regulatory requirement for toxicity studies of ECs.

In conclusion, the present work showcased how the multilayer systems toxicology framework can be applied to assess the potential toxicity of an EC liquid formulation and its aerosol. In the first-layer assessment, we determined that the toxicity of MESH Classic Tobacco and Base e-liquids was similar, and was much less than that of the TPM fraction from 3R4F CS. In the second-layer assessment, distinct molecular changes were not detected following exposure to MESH Classic Tobacco e-liquid compared with Base e-liquid treatment, as determined using HCS assays, while substantial effects were elicited by 3R4F CS TPM at much 
lower nicotine concentrations. Finally, in the third-layer assessment, we found that the impact of exposure to aerosol of the MESH Classic Tobacco or Base was markedly lower than that of exposure to 3R4F CS. This work demonstrated that the multi-layer systems toxicology framework could be useful to assess in vitro the potential-reduced impact of EC relative to $3 \mathrm{R} 4 \mathrm{~F}$ cigarettes in a comprehensive manner and is a very insightful strategy to acquire preliminary data that would be relevant to support potential clinical outcomes.

Acknowledgements The authors acknowledge Walter K. Schlage for the critical review of the manuscript. The authors would like to recognize the following individuals for their technical expertise: Francesca Caravello, Petros Kanellos, and Laurent Neau for histology; Maica Corciulo for Luminex-based analysis; Mehdi Auberson, Dariusz Peric, and Melissa Rizza for RNA extraction and quality control analysis; David Bornand and Remi Dulize for microarray processing; as well as Stephanie Boue and Fabian Moine for the preparation and publication of the dataset in the INTERVALS platform. Part of the work was presented at the Society of Toxicology Annual Meeting in March 2019 in Baltimore, MD, USA, and at the 24th Interdisciplinary Toxicology Conference in June 2019 in Vyhne, Slovakia.

Funding Philip Morris International is the sole source of funding and sponsor of this research.

\section{Compliance with ethical standards}

Conflict of interest All authors are employees of Philip Morris International.

Open Access This article is distributed under the terms of the Creative Commons Attribution 4.0 International License (http://creativeco mmons.org/licenses/by/4.0/), which permits unrestricted use, distribution, and reproduction in any medium, provided you give appropriate credit to the original author(s) and the source, provide a link to the Creative Commons license, and indicate if changes were made.

\section{References}

Bals R, Boyd J, Esposito S, Foronjy R, Hiemstra PS (2019) Electronic cigarettes: a task force report from the European Respiratory Society. Eur Respir J. https://doi.org/10.1183/13993003.01151-2018

Belcastro V, Cano S, Marescotti D et al (2019) GladiaTOX: GLobal Assessment of Dose-IndicAtor in TOXicology. Bioinformatics. https://doi.org/10.1093/bioinformatics/btz187

Benjamini Y, Hochberg Y (1995) Controlling the false discovery rate: a practical and powerful approach to multiple testing. J R Stat Soc Ser B (Methodol) 57:289-300

Boué S, Talikka M, Westra JW et al (2015) Causal biological network database: a comprehensive platform of causal biological network models focused on the pulmonary and vascular systems. Database. https://doi.org/10.1093/database/bav030

Castro NP, Osório CA, Torres C et al (2008) Evidence that molecular changes in cells occur before morphological alterations during the progression of breast ductal carcinoma. Breast Cancer Res 10(5):R87. https://doi.org/10.1186/bcr2157

Chaumont M, Pvd Borne, Bernard A et al (2019) Fourth generation e-cigarette vaping induces transient lung inflammation and gas exchange disturbances: results from two randomized clinical trials. Am J Physiol Lung Cell Mol Physiol 316(5):L705-L719. https ://doi.org/10.1152/ajplung.00492.2018

CORESTA (2015) Coresta Recommended Method No 81. Routine analytical machine for E-cigarette aerosol generation and collection-definitions and Standard Conditions. https://www.cores ta.org/sites/default/files/technical_documents/main/CRM_81.pdf. Retrieved on 11 Apr 2019

Costigan S, Lopez-Belmonte J (2017) An approach to allergy risk assessments for e-liquid ingredients. Regul Toxicol Pharmacol 87:1-8. https://doi.org/10.1016/j.yrtph.2017.04.003

Costigan S, Meredith C (2015) An approach to ingredient screening and toxicological risk assessment of flavours in e-liquids. Regul Toxicol Pharmacol 72(2):361-369. https://doi.org/10.1016/j. yrtph.2015.05.018

Cox S, Dawkins L (2018) Global and local perspectives on tobacco harm reduction: what are the issues and where do we go from here? Harm Reduct J 15(1):32. https://doi.org/10.1186/s1295 4-018-0239-5

Czégény Z, Bozi J, Sebestyén Z et al (2016) Thermal behaviour of selected flavour ingredients and additives under simulated cigarette combustion and tobacco heating conditions. J Anal Appl Pyrolysis 121:190-204. https://doi.org/10.1016/j. jaap.2016.07.020

Dai M, Wang P, Boyd AD et al (2005) Evolving gene/transcript definitions significantly alter the interpretation of GeneChip data. Nucleic Acids Res 33(20):e175. https://doi.org/10.1093/ nar/gni179

Davis MA, Eldridge S, Louden C (2013) Chapter 10-biomarkers: discovery, qualification and application. In: Wallig WMHGRA (ed) Haschek and Rousseaux's handbook of toxicologic pathology, 3rd edn. Academic Press, Boston, pp 317-352

Dinarello CA (2018) Overview of the IL-1 family in innate inflammation and acquired immunity. Immunol Rev 281(1):8-27. https ://doi.org/10.1111/imr.12621

Family Smoking Prevention and Tobacco Control Act (2009) 111 P.L. $31 ; 123$ Stat. $\S \S 1776$

Filer DL, Kothiya P, Setzer RW, Judson RS, Martin MT (2017) tcpl: the ToxCast pipeline for high-throughput screening data. Bioinformatics 33(4):618-620. https://doi.org/10.1093/bioinforma tics/btw680

Gonzalez-Suarez I, Martin F, Marescotti D et al (2016) In vitro systems toxicology assessment of a candidate modified risk tobacco product shows reduced toxicity compared to that of a conventional cigarette. Chem Res Toxicol 29(1):3-18. https:// doi.org/10.1021/acs.chemrestox.5b00321

Gonzalez-Suarez I, Marescotti D, Martin F et al (2017) In vitro systems toxicology assessment of nonflavored e-cigarette liquids in primary lung epithelial cells. Appl In Vitro Toxicol 3(1):41-55. https://doi.org/10.1089/aivt.2016.0040

Grana R, Benowitz N, Glantz SA (2014) E-cigarettes: a scientific review. Circulation 129(19):1972-1986. https://doi. org/10.1161/CIRCULATIONAHA.114.007667

Haghnegahdar A, Feng Y, Chen X, Lin J (2018) Computational analysis of deposition and translocation of inhaled nicotine and acrolein in the human body with e-cigarette puffing topographies. Aerosol Sci Technol 52(5):483-493. https://doi. org/10.1080/02786826.2018.1447644

Health Canada (1999) Determination of Tar, Water, Nicotine and Carbon Monoxide in Mainstream Tobacco Smoke. Health Canada Test Method T-11

Hoeng J, Deehan R, Pratt D et al (2012) A network-based approach to quantifying the impact of biologically active substances. Drug Discov Today 17(9-10):413-418. https://doi.org/10.1016/j. drudis.2011.11.008 
Hoeng J, Talikka M, Martin F et al (2014) Case study: the role of mechanistic network models in systems toxicology. Drug Discov Today 19(2):183-192. https://doi.org/10.1016/j.drudi s.2013.07.023

Hu W, Pasare C (2013) Location, location, location: tissue-specific regulation of immune responses. J Leukoc Biol 94(3):409-421. https://doi.org/10.1189/jlb.0413207

Iskandar AR, Gonzalez-Suarez I, Majeed S et al (2016) A framework for in vitro systems toxicology assessment of e-liquids. Toxicol Mech Methods 26(6):389-413. https://doi.org/10.3109/15376 516.2016.1170251

Iskandar AR, Mathis C, Martin F et al (2017) 3-D nasal cultures: systems toxicological assessment of a candidate modified-risk tobacco product. ALTEX Altern Anim Exp 34(1):23-48

Iskandar AR, Martin F, Leroy P et al (2018) Comparative biological impacts of an aerosol from carbon-heated tobacco and smoke from cigarettes on human respiratory epithelial cultures: a systems toxicology assessment. Food Chem Toxicol 115:109-126

Iskandar AR, Zanetti F, Kondylis A et al (2019) A lower impact of an acute exposure to electronic cigarette aerosols than to cigarette smoke in human organotypic buccal and small airway cultures was demonstrated using systems toxicology assessment. Intern Emerg Med. https://doi.org/10.1007/s11739-019-02055-x

Janovitz EB, Wallig MA (2013) Morphologic manifestation of toxic cell injury Haschek and Rousseaux's handbook of toxicologic pathology, 3rd edn. Academic Press, Boston, pp 33-36

Khatri P, Sirota M, Butte AJ (2012) Ten years of pathway analysis: current approaches and outstanding challenges. PLoS Comput Biol 8(2):e1002375. https://doi.org/10.1371/journal.pcbi.1002375

Kim B, Lee Y, Kim E et al (2013) The Interleukin-1 $\alpha$ precursor is biologically active and is likely a key alarmin in the IL-1 family of cytokines. Front Immunol 4:391. https://doi.org/10.3389/ fimmu.2013.00391

Marescotti D, Gonzalez Suarez I, Acali S et al (2016) High content screening analysis to evaluate the toxicological effects of harmful and potentially harmful constituents (HPHC). J Vis Exp. https:// doi.org/10.3791/53987

Martin F, Sewer A, Talikka M, Xiang Y, Hoeng J, Peitsch M (2014) Quantification of biological network perturbations for mechanistic insight and diagnostics using two-layer causal models. BMC Bioinformatics 15(1):238. https://doi.org/10.1186/1471-2105-15-238

McCall MN, Bolstad BM, Irizarry RA (2010) Frozen robust multiarray analysis (fRMA). Biostatistics 11(2):242-253. https://doi. org/10.1093/biostatistics/kxp059

NAS (2018) Public HePublic health consequences of e-cigarettes. The National Academies Press, Washington. https://doi.org/10.17226 124952

Notley C, Ward E, Dawkins L, Holland R (2018) The unique contribution of e-cigarettes for tobacco harm reduction in supporting smoking relapse prevention. Harm Reduct J 15(1):31

Papaseit E, Farre M, Graziano S et al (2017) Monitoring nicotine intake from e-cigarettes: measurement of parent drug and metabolites in oral fluid and plasma. Clin Chem Lab Med 55(3):415-423. https ://doi.org/10.1515/cclm-2016-0405

Rider P, Voronov E, Dinarello CA, Apte RN, Cohen I (2017) Alarmins: feel the stress. J Immunol 198(4):1395. https://doi.org/10.4049/ jimmunol.1601342
Smyth GK (2004) Linear models and empirical Bayes methods for assessing differential expression in microarray experiments. Stat Appl Genet Mol Biol 3:3. https://doi.org/10.2202/1544-6115.1027

Steiling K, Ryan J, Brody JS, Spira A (2008) The field of tissue injury in the lung and airway. Cancer Prev Res (Phila) 1(6):396-403. https://doi.org/10.1158/1940-6207.CAPR-08-0174

Stevenson M, Czekala L, Simms L, Tschierske N, Larne O, Walele T (2019) The use of Genomic Allergen Rapid Detection (GARD) assays to predict the respiratory and skin sensitising potential of e-liquids. Regul Toxicol Pharmacol RTP 103:158-165. https:// doi.org/10.1016/j.yrtph.2019.01.001

Thomson TM, Sewer A, Martin F et al (2013) Quantitative assessment of biological impact using transcriptomic data and mechanistic network models. Toxicol Appl Pharmacol. https://doi. org/10.1016/j.taap.2013.07.007 (In press)

Tierney PA, Karpinski CD, Brown JE, Luo W, Pankow JF (2016) Flavour chemicals in electronic cigarette fluids. Tobacco Control 25(e1):e10-e15. https://doi.org/10.1136/tobaccocontrol-2014052175

Wang P, Chen W, Liao J et al (2017) A device-independent evaluation of carbonyl emissions from heated electronic cigarette solvents. PLoS ONE 12(1):e0169811. https://doi.org/10.1371/journ al.pone. 0169811

WHO (2008) Overall Recommendations. In: WHO study group on tobacco produt regulation. WHO Technical Report Series: the scientific basis of tobacco product regulation. https://www.who. int/tobacco/global_interaction/tobreg/publications/9789241209 519.pdf. Retrieved on 29 Mar 2019

Xia X, Wong ST (2012) Concise review: a high-content screening approach to stem cell research and drug discovery. Stem cells (Dayton, Ohio) 30(9):1800-1807. https://doi.org/10.1002/ stem. 1168

Yan G, Du Q, Wei X et al (2018) Application of real-time cell electronic analysis system in modern pharmaceutical evaluation and analysis. Molecules (Basel Switz) 23(12):3280. https://doi. org/10.3390/molecules23123280

Zanetti F, Sewer A, Scotti E et al (2018) Assessment of the impact of aerosol from a potential modified risk tobacco product compared with cigarette smoke on human organotypic oral epithelial cultures under different exposure regimens. Food Chem Toxicol 115:148-169

Zhu SH, Lee M, Zhuang YL, Gamst A, Wolfson T (2012) Interventions to increase smoking cessation at the population level: how much progress has been made in the last two decades? Tob Control 21(2):110-118. https://doi.org/10.1136/tobaccocontrol-2011050371

Publisher's Note Springer Nature remains neutral with regard to jurisdictional claims in published maps and institutional affiliations. 